\title{
Microscopic Theory of Exciton-Exciton Annihilation in Two-Dimensional Semiconductors
}

\author{
Alexander Steinhoff and Frank Jahnke \\ Institute for Theoretical Physics, University of Bremen, 28334 Bremen, Germany ${ }^{*}$ \\ Matthias Florian \\ Institute for Theoretical Physics, University of Bremen, 28334 Bremen, Germany and \\ Department of Electrical Engineering and Computer Science, University of Michigan, Ann Arbor, MI, USA
}

\begin{abstract}
Auger-like exciton-exciton annihilation (EEA) is considered the key fundamental limitation to quantum yield in devices based on excitons in two-dimensional (2d) materials. Since it is challenging to experimentally disentangle EEA from competing processes, guidance of a quantitative theory is highly desirable. The very nature of EEA requires a material-realistic description that is not available to date. We present a many-body theory of EEA based on first-principle band structures and Coulomb interaction matrix elements that goes beyond an effective bosonic picture. Applying our theory to monolayer $\mathrm{MoS}_{2}$ encapsulated in hexagonal BN, we obtain an EEA coefficient in the order of $10^{-3} \mathrm{~cm}^{2} \mathrm{~s}^{-1}$ at room temperature, suggesting that carrier losses are often dominated by other processes, such as defect-assisted scattering. Our studies open a perspective to quantify the efficiency of intrinsic EEA processes in various $2 \mathrm{~d}$ materials in the focus of modern materials research.
\end{abstract}

\section{INTRODUCTION}

Auger recombination is a Coulomb interaction process where an electron-hole pair recombines nonradiatively by transferring the excess energy to another charge carrier. Since this process is operative at high carrier densities, it has been discussed for decades as a loss mechanism in optoelectronic devices that use highly excited semiconductors as active material [1 5].

In systems with strong Coulomb interaction, bound electron-hole pairs termed excitons can dominate the dynamics, as long as the density of excited carriers is much smaller than the Mott density [6, 7]. In this situation, Auger scattering is expected to take place between two excitons instead of three unbound particles. A prominent material class with ultra-strong Coulomb interaction are atomically thin transition metal dichalcogenides (TMDs). The discussion of Auger-like exciton-exciton annihilation (EEA) in TMDs has been started by the works of Sun et al. 8 and Kumar et al. 9. Since then, a range of EEA coefficients from several $10^{-3} \mathrm{~cm}^{2} \mathrm{~s}^{-1}$ to about $0.1 \mathrm{~cm}^{2} \mathrm{~s}^{-1}$ have been found experimentally for different TMD materials 8 14. Many of the early experiments have been performed on a $\mathrm{SiO}_{2}$ substrate. A strong reduction of exciton recombination for TMDs encapsulated in hexagonal boron nitride (hBN) 15 17] suggests that passivation plays a role, similar as for the quality of optical spectra [18. The wide spectrum of experimental results and the sensitivity of exciton lifetimes to extrinsic effects call for a theoretical prediction of intrinsic EEA coefficients to quantify their impact on device performance.

\footnotetext{
* asteinhoff@itp.uni-bremen.de

$\dagger$ Present address
}

There have been theory studies based on a two-band $k \cdot p$ model [19, Monte Carlo simulations [11] and a dipoledipole interaction model assuming spatially localized excitons 20]. While these are low-energy models, it has been pointed out in Refs. 21, 22, that EEA involves target states from higher bands. In Ref. 22, the $k \cdot p$ approach was extended to a third band, which relies on the assumption that the target states selected by energy conservation are close to the third band's extremum.

In translationally invariant systems, quantitative predictions of EEA efficiency generally require materialrealistic input to determine overlaps of Bloch states in different bands beyond high-symmetry points. The challenge is to combine the large phase space of target states with a theory for exciton-exciton interaction in second order of the Coulomb potential. A first step towards a universal description of EEA processes has been taken recently for confined semiconductor nanostructures [23, 24. Here, EEA rates have been calculated in the so-called interacting framework, which includes electronhole correlations within the initial electron-hole pairs, while neglecting those between excitons or final-state carriers.

In this paper, we present a theory of EEA in $2 \mathrm{~d}$ materials based on a many-body description of exciton-exciton scattering processes using a general band structure and Coulomb interaction matrix elements from density functional theory (DFT). It is shown that the resulting equations of motion (EOM) can not be obtained from a purely bosonic Hamiltonian with effective exciton-exciton interaction matrix elements since the fermionic substructure of excitons would be otherwise neglected. The presented approach consistently takes into account all electron-hole correlations on a two-particle level. We apply our theory to quantify EEA coefficients in monolayer $\mathrm{MoS}_{2}$ and analyze how different Bloch states contribute to the excitonexciton scattering. We also study the influence of tem- 
perature and dielectric environmental screening on EEA, finding an inverse temperature dependence that is much stronger than the dependence on substrate dielectric constants.

\section{THEORY}

Our starting point is the Hamiltonian for Bloch electrons interacting via a statically screened Coulomb potential:

$$
\begin{aligned}
H & =H_{0}+H_{\text {Coul }} \\
& =\sum_{\mathbf{k}, c} \varepsilon_{\mathbf{k}}^{c} a_{\mathbf{k}, c}^{\dagger} a_{\mathbf{k}, c}+\sum_{\mathbf{k}, v} \varepsilon_{\mathbf{k}}^{v} a_{\mathbf{k}, v}^{\dagger} a_{\mathbf{k}, v} \\
& +\frac{1}{2 \mathcal{A}} \sum_{\substack{\mathbf{k}, \mathbf{k}^{\prime}, \mathbf{q} \\
\lambda, \nu, \nu^{\prime}, \lambda^{\prime}}} V_{\mathbf{k}, \mathbf{k}^{\prime}, \mathbf{k}^{\prime}+\mathbf{q}, \mathbf{k}-\mathbf{q}}^{\lambda, \nu, \lambda^{\prime}, \lambda^{\prime}} a_{\mathbf{k}, \lambda}^{\dagger} a_{\mathbf{k}^{\prime}, \nu}^{\dagger} a_{\mathbf{k}^{\prime}+\mathbf{q}, \nu^{\prime}} a_{\mathbf{k}-\mathbf{q}, \lambda^{\prime}},
\end{aligned}
$$

where $a_{\mathbf{k}, \lambda}^{\dagger}$ and $a_{\mathbf{k}, \lambda}$ denote carrier creation and annihilation operators, respectively, $\varepsilon_{\mathbf{k}}^{c / v}$ is the energy of a carrier with momentum $\mathbf{k}$ in a conduction/valence band, $V^{\lambda, \nu, \nu^{\prime}, \lambda^{\prime}}$

, coulomb interaction matrix elements, and $\mathcal{A}$ is the crystal area. EEA emerges as a higher-order carrier-carrier interaction process within the dynamics of microscopic exciton populations, which are described by two-particle correlations (doublets) $n_{\alpha, \mathbf{q}}=\Delta\left\langle X_{\alpha, \mathbf{q}}^{\dagger} X_{\alpha, \mathbf{q}}\right\rangle$ [25]. Here, we introduced the exciton creation operator $X_{\alpha, \mathbf{q}}^{\dagger}=\sum_{\mathbf{k}, v, c} \phi_{\alpha, \mathbf{k}}^{v c}(\mathbf{q}) a_{\mathbf{k}-\mathbf{q}, \mathrm{c}}^{\dagger} a_{\mathbf{k}, \mathrm{v}}$, where $\mathbf{q}$ is the total momentum of the electron-hole pair, while $\alpha$ is the quantum number belonging to the relative motion of electron and hole. The wave functions $\phi_{\alpha, \mathbf{k}}^{v c}(\mathbf{q})$ are solutions of the Bethe-Salpeter equation (BSE) in the absence of photoexcited carriers

$$
\begin{aligned}
& \left(\varepsilon_{\mathbf{k}-\mathbf{q}}^{\mathrm{c}}-\varepsilon_{\mathbf{k}}^{\mathrm{v}}-E_{\alpha, \mathbf{q}}\right) \phi_{\alpha, \mathbf{k}}^{v c}(\mathbf{q}) \\
& -\frac{1}{\mathcal{A}} \sum_{\mathbf{k}^{\prime}, v^{\prime}, c^{\prime}} V_{\mathbf{k}-\mathbf{q}, \mathbf{k}^{\prime}, \mathbf{k}, \mathbf{k}^{\prime}-\mathbf{q}}^{c, v^{\prime}, v, c^{\prime}, \mathbf{k}^{\prime}} \phi^{v^{\prime} c^{\prime}}(\mathbf{q})=0,
\end{aligned}
$$

which comprises bound exciton states as well as unbound scattering states. Here, $E_{\alpha, \mathbf{q}}$ are the two-particle eigenenergies. We assume that the dynamics is governed by correlated electron-hole pairs, so that we can formulate a closed system of equations for the doublets $n_{\alpha, \mathbf{q}}$, while single-particle occupancies $f_{\mathbf{k}}^{\lambda}=\Delta\left\langle a_{\mathbf{k}, \lambda}^{\dagger} a_{\mathbf{k}, \lambda}\right\rangle$ are dropped. This is justified in an intermediate density regime well below the so-called Mott density, that marks a transition to a quantum phase of unbound electrons and holes 6, 7], or at low density after resonant optical excitation of bound states [26]. Since excitons are globally charge neutral, excitation-induced many-body renormalizations of the BSE eigenstates and eigenenergies can be neglected. A discussion of excitation-induced effects is given in Ref. 27].

The EOM for the $n_{\alpha, \mathbf{q}}$ including EEA are derived by applying the cluster expansion technique [25. For details, we refer to the Supporting Information (SI). The resulting hierarchy of equations is truncated by introducing a phenomenological damping $\Gamma$ of three-particle correlations and using the Markov approximation, which yields:

$$
\begin{aligned}
&\left.\frac{d}{d t} n_{\alpha, \mathbf{q}}\right|_{\mathrm{EEA}}=\frac{1}{\mathcal{A}^{2}} \sum_{\mathbf{l}} \sum_{\beta \delta} {\left[\Xi^{(\alpha, \mathbf{q}) \leftrightarrow(\beta, \mathbf{q}-\mathbf{l}),(\delta, \mathbf{l})}\right.} \\
&\left.-\Xi^{(\beta, \mathbf{q}+\mathbf{l}) \leftrightarrow(\alpha, \mathbf{q}),(\delta, \mathbf{l})}\right]
\end{aligned}
$$

with the scattering rates

$$
\begin{aligned}
& \Xi^{(\alpha, \mathbf{q}) \leftrightarrow(\beta, \mathbf{q}-\mathbf{l}),(\delta, \mathbf{l})}= \\
& \frac{2}{\hbar} \operatorname{Im}\left\{\frac{V_{\mathbf{q},-\mathbf{l}}^{D, \alpha, \beta, \delta}}{E_{\alpha, \mathbf{q}}-E_{\beta, \mathbf{q}-\mathbf{l}}-E_{\delta, \mathbf{l}}-i \Gamma} \times\right. \\
\{ & \left(V_{\mathbf{q},-\mathbf{l}}^{\alpha, \delta}\right)^{*}\left(n_{\beta, \mathbf{q}-\mathbf{l}} n_{\delta, \mathbf{l}}-n_{\alpha, \mathbf{q}}\left(n_{\delta, \mathbf{l}}+1\right)\right) \\
+ & \left.\left.\left(V_{\mathbf{q},-\mathbf{q}+\mathbf{l}}^{\alpha, \delta, \beta}\right)^{*}\left(n_{\beta, \mathbf{q}-\mathbf{l}} n_{\delta, \mathbf{l}}-n_{\alpha, \mathbf{q}}\left(n_{\beta, \mathbf{q}-\mathbf{l}}+1\right)\right)\right\}\right\}, \\
& \Xi^{(\beta, \mathbf{q}+\mathbf{l}) \leftrightarrow(\alpha, \mathbf{q}),(\delta, \mathbf{l})}= \\
& \frac{2}{\hbar} \operatorname{Im}\left\{\frac{V_{\mathbf{q}+\mathbf{l},-\mathbf{l}}^{\beta, \alpha}}{E_{\beta, \mathbf{q}+\mathbf{l}}-E_{\alpha, \mathbf{q}}-E_{\delta, \mathbf{l}}-i \Gamma} \times\right. \\
& \left(V_{\mathbf{q}+\mathbf{l},-\mathbf{l}}^{\beta, \alpha, \delta}\right)^{*}\left(n_{\alpha, \mathbf{q}} n_{\delta, \mathbf{l}}-n_{\beta, \mathbf{q}+\mathbf{l}}\left(n_{\delta, \mathbf{l}}+1\right)\right) \\
+ & \left.\left.\left(V_{\mathbf{q}+\mathbf{l},-\mathbf{q}}^{\beta, \delta}\right)^{*}\left(n_{\alpha, \mathbf{q}} n_{\delta, \mathbf{l}}-n_{\beta, \mathbf{q}+\mathbf{l}}\left(n_{\alpha, \mathbf{q}}+1\right)\right)\right\}\right\} .
\end{aligned}
$$

EEA is a second-order process in terms of effective exciton-exciton interaction matrix elements

$$
\begin{aligned}
& V_{\mathbf{q},-1}^{\alpha, \beta}=V_{\mathbf{q},-1}^{D, \alpha, \beta, \delta}-V_{\mathbf{q},-1}^{X, \alpha, \beta, \delta}, \\
& V_{\mathbf{q},-\mathbf{l}}^{D / X, \alpha, \beta, \delta}=V_{\alpha, \beta, \delta, \mathbf{q},-\mathbf{l}}^{(1), D / X}-V_{\alpha, \beta, \delta, \mathbf{q}-\mathbf{l},-\mathbf{l}}^{(2), D / X}, \\
& V_{\alpha, \beta, \delta, \mathbf{q},-1}^{(1), D}= \\
& \sum_{\mathbf{k}, \mathbf{p}} \sum_{v, c, c^{\prime}, v^{\prime \prime}, c^{\prime \prime}} \phi_{\alpha, \mathbf{k}}^{v, c}(\mathbf{q})\left(\phi_{\delta, \mathbf{p}+\mathbf{l}}^{v^{\prime \prime}, c^{\prime \prime}}(\mathbf{l})\right)^{*}\left(\phi_{\beta, \mathbf{k}}^{v, c^{\prime}}(\mathbf{q}-\mathbf{l})\right)^{*} \times \\
& \begin{array}{l}
V_{\mathbf{k}}^{c^{\prime}, c^{\prime \prime}, v^{\prime \prime}, c}, \mathbf{l}, \mathbf{p}, \mathbf{p}+\mathbf{l}, \mathbf{k}-\mathbf{q} \\
\end{array} \\
& V_{\alpha, \beta, \delta, \mathbf{q},-1}^{(1), X}= \\
& \sum_{\mathbf{k}, \mathbf{p}} \sum_{v, c, c^{\prime}, v^{\prime \prime}, c^{\prime \prime}} \phi_{\alpha, \mathbf{k}}^{v, c}(\mathbf{q})\left(\phi_{\delta, \mathbf{p}+\mathbf{l}}^{v^{\prime \prime}, c^{\prime \prime}}(\mathbf{l})\right)^{*}\left(\phi_{\beta, \mathbf{k}}^{v, c^{\prime}}(\mathbf{q}-\mathbf{l})\right)^{*} \times
\end{aligned}
$$

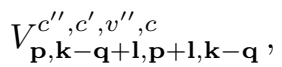

$$
\begin{aligned}
& V_{\alpha, \beta, \delta, \mathbf{q},-1}^{(2), D}= \\
& \sum_{\mathbf{k}, \mathbf{p}} \sum_{v, c, v^{\prime}, v^{\prime \prime}, c^{\prime \prime}}\left(\phi_{\beta, \mathbf{k}}^{v, c}(\mathbf{q})\right)^{*}\left(\phi_{\delta, \mathbf{p}+\mathbf{l}}^{v^{\prime \prime}, c^{\prime \prime}}(\mathbf{l})\right)^{*} \phi_{\alpha, \mathbf{k}+\mathbf{l}}^{v^{\prime}, c}(\mathbf{q}+\mathbf{l}) \times \\
& V_{\mathbf{k}+\mathbf{l}, \mathbf{p}, \mathbf{p}+\mathbf{1}, \mathbf{k}}^{v^{\prime}, v^{\prime \prime}}, \\
& V_{\alpha, \beta, \delta, \mathbf{q},-1}^{(2), X}= \\
& \sum_{\mathbf{k}, \mathbf{p}} \sum_{v, c, v^{\prime}, v^{\prime \prime}, c^{\prime \prime}}\left(\phi_{\beta, \mathbf{k}}^{v, c}(\mathbf{q})\right)^{*}\left(\phi_{\delta, \mathbf{p}+\mathbf{l}}^{v^{\prime \prime}, c^{\prime \prime}}(\mathbf{l})\right)^{*} \phi_{\alpha, \mathbf{k}+\mathbf{l}}^{v^{\prime}, c}(\mathbf{q}+\mathbf{l}) \times \\
& V_{\mathbf{p}, \mathbf{k}+\mathbf{l}, \mathbf{p}+\mathbf{l}, \mathbf{k}}^{c^{\prime \prime}, v^{\prime},}
\end{aligned}
$$




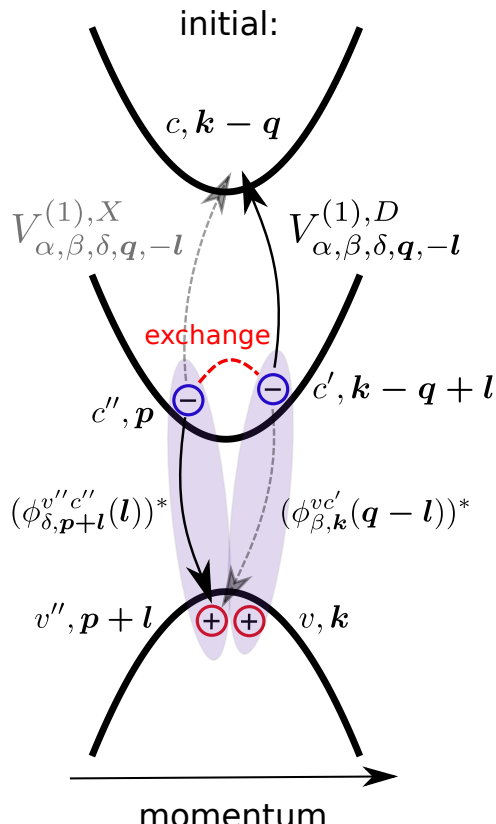

momentum

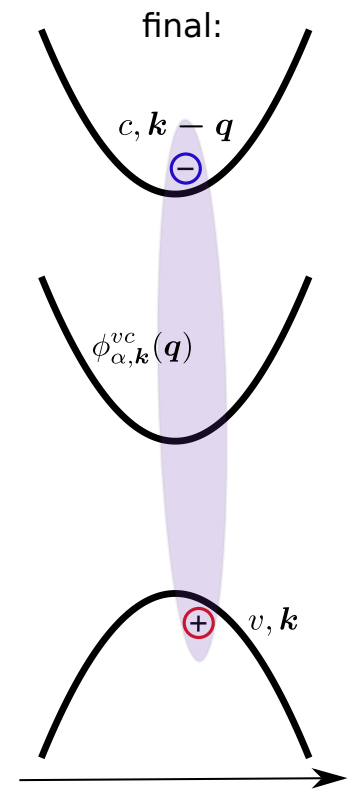

momentum

FIG. 1. Illustration of an electron-mediated EEA process described by effective exciton-exciton interaction matrix elements $V_{\alpha, \beta, \delta, \mathbf{q},-1}^{(1), D / X}$. In the direct (D) process depicted by solid black arrows, an exciton in state $|\delta, \mathbf{l}\rangle$ is annihilated by means of inter-gap electron scattering. Excess momentum and energy are transferred to a second electron that is part of an exciton in state $|\beta, \mathbf{q}-\mathbf{l}\rangle$, which is thereby excited into state $|\alpha, \boldsymbol{q}\rangle$. In the exchange-like (X) process shown by dashed grey arrows, electrons in states $\left|c^{\prime \prime}, \boldsymbol{p}\right\rangle$ and $\left|c^{\prime}, \boldsymbol{k}-\boldsymbol{q}+\boldsymbol{l}\right\rangle$ are exchanged between the excitons, leading to the same final state. Since Coulomb interaction conserves spin, the direct process is allowed for like-spin excitons $|\delta, \mathbf{l}\rangle$ only.

where $V^{(1)}$ and $V^{(2)}$ describe Auger-like scattering of electrons and holes, respectively. Hence the effective interaction is composed of elementary scattering processes weighted by the respective two-particle wave functions, where the relative minus sign between $V^{(1)}$ and $V^{(2)}$ reflects the opposite charges of electrons and holes. This is similar in exciton-phonon interaction [28]. An elementary electron scattering process belonging to the rate $\Xi^{(\alpha, \mathbf{q}) \leftrightarrow(\beta, \mathbf{q}-\mathbf{l}),(\delta, \mathbf{l})}$ is schematically shown in Fig. 1. Energy conservation is softened by the finite exciton lifetimes.

Note that the EOM do not have the form of a Boltzmann equation. Accordingly, as we demonstrate in the SI, the equations can not be derived from an exciton Hamiltonian with effective exciton-exciton interaction and bosonic commutation relations. This is due to the fact that excitons are composite particles with a fermionic substructure that can not be captured in a purely bosonic picture. In our theory, additional scattering terms emerge from the exchange of electrons or holes between two excitons, as symbolized by the dashed red line in Fig. 1. On the other hand, a bosonic theory can only account for exchange of entire excitons. It has been

pointed out in more general terms by M. Combescot et al. that it is not possible to formulate a closed expression for an effective exciton-exciton interaction potential [29]. Although we have introduced exciton-exciton interaction matrix elements (5), these can not be interpreted as such an effective interaction potential, since the very structure of the EOM (3) is beyond a purely bosonic picture. An alternative approach to treat excitons as non-ideal bosons is to introduce fermionic corrections into the commutator of exciton operators [30. As we show in the Appendix, our theory can only be mapped to an effective bosonic Hamiltonian for an exciton distribution close to equilibrium if the exchange of whole excitons is neglected. If fermionic exchange effects are included in the effective matrix elements, EEA efficiency in encapsulated $\mathrm{MoS}_{2}$ is overestimated by a factor 2 . This is partly remedied by neglecting fermionic exchange as well due to a compensation between the different exchange effects.

The EOM are completed by a phenomenological excitonphonon scattering term in relaxation-time approximation that accounts for relaxation and cooling of the exciton gas:

$$
\left.\frac{d}{d t} n_{\alpha, \mathbf{q}}\right|_{\text {relax }}=\frac{N_{\alpha, \mathbf{q}}(T)-n_{\alpha, \mathbf{q}}}{\tau_{\text {relax }}}
$$

with Bose functions $N_{\alpha, \mathbf{q}}(T)$. We choose $\tau_{\text {relax }}=50 \mathrm{fs}$ as relaxation time [28]. Since exciton-phonon scattering is several orders of magnitude faster than EEA, we assume that a microscopic description of exciton-phonon coupling would not improve our results.

We combine our theory of EEA with band structures and screened Coulomb matrix elements on a DFT level as input for Eqs. (2), (5) and (3). Details on the DFT calculation are provided in the SI. The main effect of a GW correction would be an increased band gap, which is reduced again due to environmental screening [31]. We mimic this effect by artificially increasing the band gap such that the bright exciton energy is $E_{1 \mathrm{~s} \text {,bright }}=1900$ $\mathrm{meV}$ [18. As an interface between first-principle and excited-carrier theory we utilize a lattice Hamiltonian formulated in a localized basis of Wannier orbitals $\left(\mathrm{d}_{z^{2}}\right.$, $\mathrm{d}_{x z}, \mathrm{~d}_{y z}, \mathrm{~d}_{x^{2}-y^{2}}$ and $\mathrm{d}_{x y}$ for Mo, $\mathrm{p}_{x}, \mathrm{p}_{y}$ and $\mathrm{p}_{z}$ for $\mathrm{S}$ ). Spin-orbit interaction is included using an on-site $\boldsymbol{L} \cdot \boldsymbol{S}$ coupling Hamiltonian. From the band structure obtained by diagonalization of the lattice Hamiltonian we consider two valence bands and three conduction bands for each spin degree of freedom, which is sufficient to capture all scattering states at about twice the 1s-exciton energy involved in the annihilation of 1s-excitons. Coulomb matrix elements including environmental screening effects are parametrized as a function of $|\mathbf{q}|$ using the Wannier function continuum electrostatics approach 32 in the localized basis. Unless stated otherwise, we assume a dielectric environment given by $\mathrm{hBN}$ encapsulation layers with a dielectric constant of $\varepsilon_{\mathrm{hBN}}=\sqrt{4.95 \cdot 2.86}$ [3]. In addition a narrow gap of $0.3 \mathrm{~nm}$ between the monolayer and the surrounding hBN layers has been taken into account 34. 
The numerical simulation of EEA is constrained by the high-dimensionality of the problem. In the following, we focus on Bloch states in the K-valley, where bright excitons are located in monolayer $\mathrm{MoS}_{2}$. An explicit treatment of excitons in the equivalent $\mathrm{K}^{\prime}$-valley is not necessary assuming that Auger-like EEA involving carrier scattering between $\mathrm{K}$ - and $\mathrm{K}^{\prime}$-valley is inefficient due to the large momentum transfer it involves. Numerical convergence of the results is discussed in the SI.

\section{RESULTS}

As a first step, we diagonalize the BSE (2) to obtain the two-particle spectrum and wave functions, finding a bright 1s-exciton binding energy of $287 \mathrm{meV}$. The EOM for exciton populations are solved including EEA (3) and relaxation (6) contributions. To this end, we assume that an incoherent exciton gas with a density of $10^{12} \mathrm{~cm}^{-2}$ has formed from optically excited electron-hole pairs due to ultrafast exciton-phonon interaction [26] before EEA sets in. A phenomenological damping $\Gamma=50 \mathrm{meV}$ is used. As we show in the SI, the dependence of our results on $\Gamma$ is weak. The efficiency of EEA is quantified by analyzing the time dependence of the total exciton density $n_{\mathrm{X}}=\frac{1}{A} \sum_{\alpha, \mathbf{q}} n_{\alpha, \mathbf{q}}$ as shown in Fig. 2(b). Since relaxation of excitons is much faster than EEA, the exciton distribution is close to equilibrium at all times. The overall behavior of the exciton density is therefore captured very well by a macroscopic differential equation that has been used before to discuss EEA qualitatively []ㅜㄹ

$$
\frac{d}{d t} n_{\mathrm{X}}=-k_{\mathrm{EEA}} n_{\mathrm{X}}^{2}
$$

with the solution $n_{\mathrm{X}}(t)=n_{\mathrm{X}, 0}\left(1+n_{\mathrm{X}, 0} k_{\mathrm{EEA}} t\right)^{-1}$. We use the solution as a fit formula to extract the EEA coefficient $k_{\mathrm{EEA}}$ that can be conveniently compared to experiment. The numerically converged value is estimated as $k_{\mathrm{EEA}}=2.8 \times 10^{-3} \mathrm{~cm}^{2} \mathrm{~s}^{-1}$.

In Fig. 2(c), we analyze the microscopic contribution of different Bloch states to the EEA dynamics in terms of the scattering rates between two-particle states $|\alpha, \mathbf{q}\rangle$, $|\delta, \mathbf{l}\rangle$ on one side and $|\beta, \mathbf{q}+\mathbf{l}\rangle$ on the other side at time $t=0$ as defined in Eq. (3). The dominant scattering channel involves the 1s-exciton states $|\alpha, \mathbf{q}\rangle,|\delta, \mathbf{l}\rangle$ as initial states and high-energy states $|\beta, \mathbf{q}+\mathbf{l}\rangle$ as final states. As a benchmark, we compute the weighted quantities

$$
\begin{aligned}
& \Lambda_{\mathbf{k}}^{\mathrm{i}, c}=\sum_{\alpha, \beta, \delta, \mathbf{l}, \mathbf{q}^{\prime}, v}\left|\phi_{\alpha, \mathbf{k}+\mathbf{q}^{\prime}}^{v, c}\left(\mathbf{q}^{\prime}\right)\right|^{2} \Xi^{\left(\beta, \mathbf{q}^{\prime}+\mathbf{l}\right) \leftrightarrow\left(\alpha, \mathbf{q}^{\prime}\right),(\delta, \mathbf{l})}, \\
& \Lambda_{\mathbf{k}}^{\mathrm{i}, v}=\sum_{\alpha, \beta, \delta, \mathbf{l}, \mathbf{q}^{\prime}, c}\left|\phi_{\alpha, \mathbf{k}}^{v, c}\left(\mathbf{q}^{\prime}\right)\right|^{2} \Xi^{\left(\beta, \mathbf{q}^{\prime}+\mathbf{l}\right) \leftrightarrow\left(\alpha, \mathbf{q}^{\prime}\right),(\delta, \mathbf{l})}, \\
& \Lambda_{\mathbf{k}}^{\mathrm{f}, c}=\sum_{\alpha, \beta, \delta, \mathbf{q}, \mathbf{q}^{\prime}, v}\left|\phi_{\beta, \mathbf{k}+\mathbf{q}^{\prime}}^{v, c}\left(\mathbf{q}^{\prime}\right)\right|^{2} \Xi^{\left(\beta, \mathbf{q}^{\prime}\right) \leftrightarrow(\alpha, \mathbf{q}),\left(\delta, \mathbf{q}^{\prime}-\mathbf{q}\right)}, \\
& \Lambda_{\mathbf{k}}^{\mathrm{f}, v}=\sum_{\alpha, \beta, \delta, \mathbf{q}, \mathbf{q}^{\prime}, c}\left|\phi_{\beta, \mathbf{k}}^{v, c}\left(\mathbf{q}^{\prime}\right)\right|^{2} \Xi^{\left(\beta, \mathbf{q}^{\prime}\right) \leftrightarrow(\alpha, \mathbf{q}),\left(\delta, \mathbf{q}^{\prime}-\mathbf{q}\right)}
\end{aligned}
$$
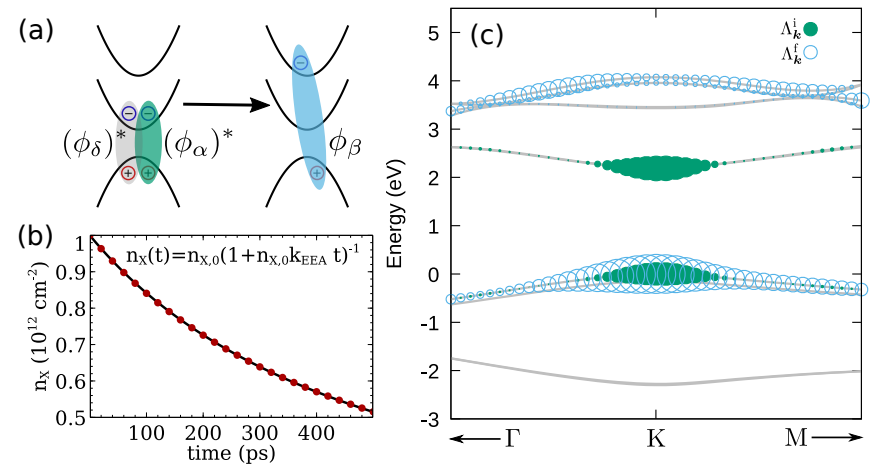

FIG. 2. (a) Fundamental EEA process: An exciton in state $|\delta\rangle$ is annihilated, while a second exciton is promoted from low-energy state $|\alpha\rangle$ to high-energy state $|\beta\rangle$. (b) Time dependence of the total exciton density $n_{\mathrm{X}}$ in $\mathrm{hBN}$-encapsulated $\mathrm{MoS}_{2}$ at $T=300 \mathrm{~K}$. Red dots correspond to the numerical solution of the EOM (3) and (6), while the analytic solution of the simplified ODE (7) is shown as a black solid line. (c) Contribution of Bloch bands to EEA scattering rates. The symbol size represents the projection of initial (full green circles) and final (open blue circles) two-particle states onto Bloch states according to the corresponding two-particle wave functions, as described by the quantities $\Lambda_{\mathbf{k}}^{\mathrm{i}}$ and $\Lambda_{\mathbf{k}}^{\mathrm{f}}$ defined in Eq. [8]. The single-particle band structure is shown in grey.

corresponding to the contributions of conduction- and valence-band states to the initial (i) and final (f) states. The rates $\Xi$ microscopically determine the scattering efficiency out of state $\left|\alpha, \mathbf{q}^{\prime}\right\rangle$ and into state $\left|\beta, \mathbf{q}^{\prime}\right\rangle$, respectively, according to population factors, energy conservation and Coulomb matrix elements. The modulus square of two-particle wave functions is then used heuristically to project the rates onto the single-particle Bloch states. Contributions to the initial state are concentrated around the fundamental band gap. Remarkably, all contributions to the final state stem from the topmost valence band and the fifth and sixth conduction bands, which belong to the subspace of bands with $\mathrm{d}_{m= \pm 2}$-orbital character. This is consistent with the discussion in Ref. [21, 22. Hence, EEA in monolayer $\mathrm{MoS}_{2}$ is essentially driven by electron-assisted processes. Moreover, we find that final electron states apart from the K-point are favored, which justifies our material-realistic approach.

Fig. 3 shows the dependence of EEA in monolayer $\mathrm{MoS}_{2}$ on temperature and dielectric environmental screening. We find that $k_{\mathrm{EEA}}$ is inversely proportional to temperature. The only effect of temperature in our theory is the width of quasi-thermal Bose functions to which exciton distributions relax by virtue of the phenomenological term (6). We therefore deduce that an increased population of exciton states with small momentum at low temperatures is favorable for EEA. As Fig. 3(b) shows, the efficiency of EEA decreases with increasing dielectric constant of the environment, which is expected due to the Coulomb nature of Auger scattering. However, the dependence is rather weak given the quadratic dependence of scattering rates on the screened Coulomb interaction. 

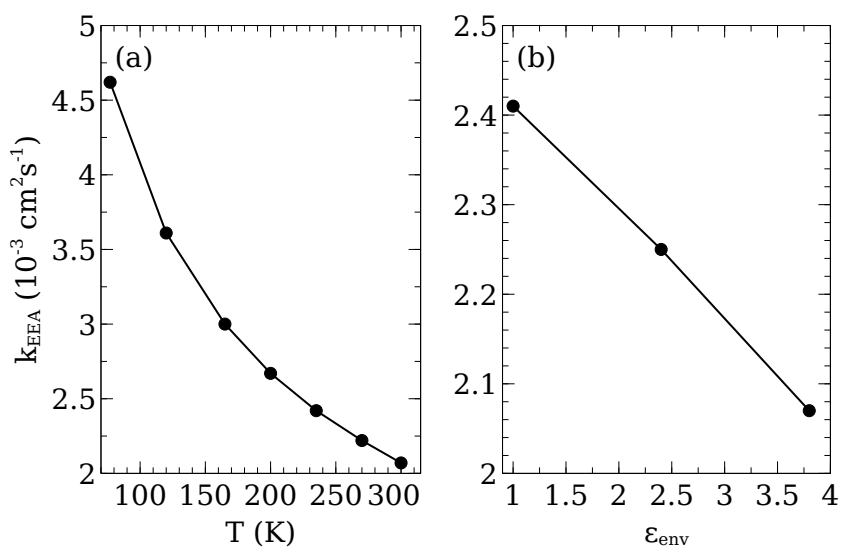

FIG. 3. (a) Temperature dependence of EEA coefficient in hBN-encapsulated $\mathrm{MoS}_{2}$. (b) Dependence of EEA coefficient for $\mathrm{MoS}_{2}$ on average dielectric constant of environment $\varepsilon_{\mathrm{env}}=$ $\left(\varepsilon_{\text {top }}+\varepsilon_{\text {bottom }}\right) / 2$ at $T=300 \mathrm{~K}$.

We attribute this to Auger-like EEA relying to a large degree on scattering processes with large momentum transfer as underlined by Fig. 2(c). Environmental screening is most efficient at small momenta, while screening at large momenta is determined by the polarization of the TMD monolayer itself [32. Our results are in line with the weak dependence on the dielectric environmental reported in Ref. 35, while a stronger dependence has been found in Ref. [36. We infer that surface chemistry, not dielectric screening, is the decisive factor.

In various experiments, EEA coefficients have been extracted from time-resolved photoluminescence (PL) measurements. For a comparison between experiment and theory, one has to keep in mind that we limited ourselves to EEA in a single valley. Inter-valley excitons consisting of electrons and holes from different valleys are approximately degenerate to intra-valley excitons. Thus, if exciton relaxation is much faster than EEA, half of the exciton density occupies inter-valley states, which can only decay assisted by slow inter-valley scattering due to momentum conservation. Our calculation therefore overestimates the fraction of excitons decaying via EEA by a factor 2 (corresponding to state $|\delta\rangle$ in Fig. 2(a)), while the assisting scattering process can be provided by all excitons $(|\alpha\rangle$ in Fig. 2(a)). Hence, according to Eq. (7), the coefficients we calculate have to be divided by 2 to be compared to experiment, which yields $k_{\mathrm{EEA}}=1.4 \times 10^{-3}$ $\mathrm{cm}^{2} \mathrm{~s}^{-1}$ including $\mathrm{k}$-mesh convergence.

The EEA coefficient we find is smaller than those reported in literature. The smallest coefficients $k_{\mathrm{EEA}}=$ $6 \times 10^{-3} \mathrm{~cm}^{2} \mathrm{~s}^{-1}[15]$ and $k_{\mathrm{EEA}}=4 \times 10^{-3} \mathrm{~cm}^{2} \mathrm{~s}^{-1}$ [17] for $\mathrm{WS}_{2}$ encapsulated in $\mathrm{hBN}$ are in the same order of magnitude, while the coefficient drastically increases when the $\mathrm{WS}_{2}$ is in direct contact with a $\mathrm{SiO}_{2}$ substrate. Since $\mathrm{SiO}_{2}$-supported TMD layers show strong defect activity e.g. in PL [18] and STM [37, it is plausible that defect-assisted processes dominate the dynamics in such samples. This is corroborated by the fact that lumines- cence efficiency of monolayer $\mathrm{MoS}_{2}$ can be drastically increased by a chemical treatment eliminating defectmediated nonradiative recombination 38. Furthermore, it has been shown that defect-assisted exciton annihiliation has a contribution that is quadratic in exciton density [39], just as EEA.

There is a number of intrinsic processes that provide additional channels for exciton decay, which implies that our theory sets a lower limit to observable EEA coefficients. We have already discussed that inter-valley scattering would provide decay channels for inter-valley excitons. Also, phonon-assisted processes are expected to foster annihilation of excitons with large momentum [21. Radiative decay of uncorrelated electron-hole pairs depends quadratically on carrier density [40. Since a certain fraction of carriers is always ionized, these processes can distort the EEA coefficient.

We finally discuss possible variations of EEA coefficients due to uncertainties of the underlying first-principle calculation. In the SI, we show how the coefficient depends on $E_{1 \mathrm{~s} \text {,bright }}$, which determines the final-state DOS at about $2 E_{1 \mathrm{~s}, \text { bright }}(|\beta\rangle$ in $2(\mathrm{a}))$. For example, higher energies of the third conduction band can be simulated by a lower exciton energy. We find that a variation of \pm 50 $\mathrm{meV}$ in $E_{1 \mathrm{~s} \text {,bright }}$ changes the results by $\pm 30 \%$. Moreover, the used first-principle method has an effect on the position of side valleys relative to the K-valley [41. The influence of relative valley positions has been studied by comparing TMD multilayer structures. EEA efficiency decreases with increasing layer number in materials where indirect excitons become dominant [10], while the trend is weaker as long as the material remains direct [13. We therefore expect that intrinsic EEA is less efficient in tungsten-based TMD monolayers than in $\mathrm{MoS}_{2}$.

\section{CONCLUSION}

In conclusion, our microscopic theory of EEA allows, for the first time, to quantify the efficiency of intrinsic EEA, which is often masked by extrinsic processes in experiment. The result of $k_{\mathrm{EEA}} \approx 10^{-3} \mathrm{~cm}^{2} \mathrm{~s}^{-1}$ for hBNencapsulated monolayer $\mathrm{MoS}_{2}$ is consistent with observation. As we have shown, the physics of EEA can not be captured by an effective bosonic theory. In the future, the theory can be applied to various members of the expanding family of $2 \mathrm{~d}$ materials, such as perovskites [42, black phosphorus [43, 44] and TMD hetero-bilayers hosting spatially indirect excitons of dipolar nature [45, 46].

\section{Acknowledgement}

We acknowledge financial support from the Deutsche Forschungsgemeinschaft (RTG 2247 "Quantum Mechanical Materials Modelling") as well as resources for computational time at the HLRN (Hannover/Berlin). M.F. acknowledges support by the Alexander von Humboldt foundation. The authors would also like to thank Prof. M. Kira for valuable discussions. 
[1] B. Sermage, D. Chemla, D. Sivco, and A. Cho, Comparison of auger recombination in GaInAs-AlInAs multiple quantum well structure and in bulk GaInAs, IEEE Journal of Quantum Electronics 22, 774 (1986).

[2] A. Haug, Auger recombination in quantum well semiconductors: calculation with realistic energy bands, Semiconductor Science and Technology 7, 1337-1340 (1992)

[3] G. Fuchs, C. Schiedel, A. Hangleiter, V. Härle, and F. Scholz, Auger recombination in strained and unstrained InGaAs/InGaAsP multiple quantum-well lasers, Applied Physics Letters 62, 396 (1993).

[4] V. I. Klimov, A. A. Mikhailovsky, D. W. McBranch, C. A. Leatherdale, and M. G. Bawendi, Quantization of multiparticle auger rates in semiconductor quantum dots, Science 287, 1011 (2000)

[5] G. Dukovic, E. Knoesel, L. E. Brus, T. F. Heinz, and F. Wang, Observation of rapid auger recombination in optically excited semiconducting carbon nanotubes, Physical Review B 70, 241403 (2004).

[6] D. Semkat, F. Richter, D. Kremp, G. Manzke, W.-D. Kraeft, and K. Henneberger, Ionization equilibrium in an excited semiconductor: Mott transition versus boseeinstein condensation, Physical Review B 80, 155201 (2009)

[7] A. Steinhoff, M. Florian, M. Rösner, G. Schönhoff, T. O. Wehling, and F. Jahnke, Exciton fission in monolayer transition metal dichalcogenide semiconductors, Nature Communications 8, 1166 (2017).

[8] D. Sun, Y. Rao, G. A. Reider, G. Chen, Y. You, L. Brézin, A. R. Harutyunyan, and T. F. Heinz, Observation of rapid Exciton-Exciton annihilation in monolayer molybdenum disulfide, Nano Letters 14, 5625 (2014).

[9] N. Kumar, Q. Cui, F. Ceballos, D. He, Y. Wang, and H. Zhao, Exciton-exciton annihilation in $\mathrm{MoSe}_{2}$ monolayers, Physical Review B 89, 125427 (2014).

[10] L. Yuan and L. Huang, Exciton dynamics and annihilation in WS2 2D semiconductors, Nanoscale 7, 7402 (2015)

[11] S. Mouri, Y. Miyauchi, M. Toh, W. Zhao, G. Eda, and K. Matsuda, Nonlinear photoluminescence in atomically thin layered $\mathrm{WSe}_{2}$ arising from diffusion-assisted excitonexciton annihilation, Physical Review B 90, 155449 (2014)

[12] C. Poellmann, P. Steinleitner, U. Leierseder, P. Nagler, G. Plechinger, M. Porer, R. Bratschitsch, C. Schüller, T. Korn, and R. Huber, Resonant internal quantum transitions and femtosecond radiative decay of excitons in monolayer WSe 2, Nature Materials 14, 889 (2015).

[13] S. Sim, D. Lee, J. Lee, M. Cha, S. Cha, W. Heo, S. Cho, W. Shim, K. Lee, J. Yoo, R. P. Prasankumar, H. Choi, and M.-H. Jo, Role of weak interlayer coupling in ultrafast exciton-exciton annihilation in two-dimensional rhenium dichalcogenides, Physical Review B 101, 174309 (2020).

[14] R. Perea-Causín, S. Brem, R. Rosati, R. Jago, M. Kulig, J. D. Ziegler, J. Zipfel, A. Chernikov, and E. Malic, Exciton propagation and halo formation in two-dimensional materials, Nano Letters 19, 7317 (2019)

[15] Y. Hoshi, T. Kuroda, M. Okada, R. Moriya, S. Masubuchi, K. Watanabe, T. Taniguchi, R. Kitaura, and T. Machida, Suppression of exciton-exciton annihilation in tungsten disulfide monolayers encapsulated by hexagonal boron nitrides, Physical Review B 95, 241403 (2017).

[16] D. F. Cordovilla Leon, Z. Li, S. W. Jang, and P. B. Deotare, Hot exciton transport in $\mathrm{WSe}_{2}$ monolayers, Physical Review B 100, 241401 (2019)

[17] J. Zipfel, M. Kulig, R. Perea-Causín, S. Brem, J. D. Ziegler, R. Rosati, T. Taniguchi, K. Watanabe, M. M. Glazov, E. Malic, and A. Chernikov, Exciton diffusion in monolayer semiconductors with suppressed disorder, Physical Review B 101, 115430 (2020)

[18] F. Cadiz, E. Courtade, C. Robert, G. Wang, Y. Shen, H. Cai, T. Taniguchi, K. Watanabe, H. Carrere, D. Lagarde, M. Manca, T. Amand, P. Renucci, S. Tongay, X. Marie, and B. Urbaszek, Excitonic linewidth approaching the homogeneous limit in $\mathrm{MoS}_{2}$-Based van der waals heterostructures, Physical Review X 7, 021026 (2017)

[19] S. Konabe and S. Okada, Effect of coulomb interactions on optical properties of monolayer transition-metal dichalcogenides, Physical Review B 90, 155304 (2014).

[20] E. Chatterjee, D. B. S. Soh, C. Rogers, D. J. Gray, and H. Mabuchi, Low-temperature annihilation rate for quasilocalized excitons in monolayer $\mathrm{MoS}_{2}$, Physical Review B 100, 155405 (2019).

[21] M. Danovich, V. Zólyomi, V. I. Fal'ko, and I. L. Aleiner, Auger recombination of dark excitons in WS 2 and WSe 2 monolayers, 2D Materials 3, 035011 (2016)

[22] B. Han, C. Robert, E. Courtade, M. Manca, S. Shree, T. Amand, P. Renucci, T. Taniguchi, K. Watanabe, X. Marie, L. Golub, M. Glazov, and B. Urbaszek, Exciton states in monolayer $\mathrm{MoSe}_{2}$ and $\mathrm{MoTe}_{2}$ probed by upconversion spectroscopy, Physical Review X 8, 031073 (2018)

[23] J. P. Philbin and E. Rabani, Electron-Hole correlations govern auger recombination in nanostructures, Nano Letters 18, 7889 (2018)

[24] J. P. Philbin, A. Brumberg, B. T. Diroll, W. Cho, D. V. Talapin, R. D. Schaller, and E. Rabani, Area and thickness dependence of auger recombination in nanoplatelets, The Journal of Chemical Physics 153, 054104 (2020).

[25] M. Kira and S. W. Koch, Many-body correlations and excitonic effects in semiconductor spectroscopy, Progress in Quantum Electronics 30, 155 (2006)

[26] M. Selig, G. Berghäuser, M. Richter, R. Bratschitsch, A. Knorr, and E. Malic, Dark and bright exciton formation, thermalization, and photoluminescence in monolayer transition metal dichalcogenides, 2D Materials 5, 035017 (2018)

[27] A. Schleife, C. Rödl, F. Fuchs, K. Hannewald, and F. Bechstedt, Optical absorption in degenerately doped semiconductors: Mott transition or mahan excitons?, Physical Review Letters 107, 236405 (2011).

[28] M. Selig, G. Berghäuser, A. Raja, P. Nagler, C. Schüller, T. F. Heinz, T. Korn, A. Chernikov, E. Malic, and A. Knorr, Excitonic linewidth and coherence lifetime in monolayer transition metal dichalcogenides, Nature Communications 7, 13279 (2016)

[29] M. Combescot and O. Betbeder-Matibet, The effective bosonic hamiltonian for excitons reconsidered, Europhysics Letters 58, 87-92 (2002).

[30] F. Katsch, M. Selig, A. Carmele, and A. Knorr, The- 
ory of Exciton-Exciton interactions in monolayer transition metal dichalcogenides, physica status solidi (b) 255, 1800185 (2018)

[31] K. S. Thygesen, Calculating excitons, plasmons, and quasiparticles in $2 \mathrm{D}$ materials and van der waals heterostructures, 2D Materials 4, 022004 (2017)

[32] M. Rösner, E. Şaşıŏlu, C. Friedrich, S. Blügel, and T. O. Wehling, Wannier function approach to realistic coulomb interactions in layered materials and heterostructures, Physical Review B 92, 085102 (2015).

[33] L. Artús, R. Cuscó, T. Taniguchi, G. Cassabois, B. Gil, and A. Segura, Natural optical anisotropy of h-BN: highest giant birefringence in a bulk crystal through the midinfrared to ultraviolet range, Physical Review Materials 2, 024001 (2018)

[34] M. Florian, M. Hartmann, A. Steinhoff, J. Klein, A. W. Holleitner, J. J. Finley, T. O. Wehling, M. Kaniber, and C. Gies, The dielectric impact of layer distances on exciton and trion binding energies in van der waals heterostructures, Nano Letters 18, 2725 (2018)

[35] Y. Yu, Y. Yu, C. Xu, A. Barrette, K. Gundogdu, and L. Cao, Fundamental limits of exciton-exciton annihilation for light emission in transition metal dichalcogenide monolayers, Physical Review B 93, 201111 (2016).

[36] A. J. Goodman, D.-H. Lien, G. H. Ahn, L. L. Spiegel, M. Amani, A. P. Willard, A. Javey, and W. A. Tisdale, Substrate-dependent exciton diffusion and annihilation in chemically treated MoS2 and WS2, The Journal of Physical Chemistry C 124, 12175 (2020).

[37] J. Klein, A. Kerelsky, M. Lorke, M. Florian, F. Sigger, J. Kiemle, M. C. Reuter, T. Taniguchi, K. Watanabe, J. J. Finley, A. N. Pasupathy, A. W. Holleitner, F. M. Ross, and U. Wurstbauer, Impact of substrate induced band tail states on the electronic and optical properties of MoS2, Applied Physics Letters 115, 261603 (2019).

[38] M. Amani, D.-H. Lien, D. Kiriya, J. Xiao, A. Azcatl, J. Noh, S. R. Madhvapathy, R. Addou, S. KC, M. Dubey, K. Cho, R. M. Wallace, S.-C. Lee, J.-H. He, J. W. Ager, X. Zhang, E. Yablonovitch, and A. Javey, Near-unity photoluminescence quantum yield in MoS2, Science 350, 1065 (2015)

[39] H. Wang, J. H. Strait, C. Zhang, W. Chan, C. Manolatou, S. Tiwari, and F. Rana, Fast exciton annihilation by capture of electrons or holes by defects via auger scattering in monolayer metal dichalcogenides, Physical Review B 91, 165411 (2015)

[40] M. Kira, F. Jahnke, W. Hoyer, and S. W. Koch, Quantum theory of spontaneous emission and coherent effects in semiconductor microstructures, Progress in Quantum Electronics 23, 189 (1999).

[41] H. Shi, H. Pan, Y.-W. Zhang, and B. I. Yakobson, Quasiparticle band structures and optical properties of strained monolayer MoS2 and WS2, Physical Review B 87, 155304 (2013).

[42] J.-X. Shen, X. Zhang, S. Das, E. Kioupakis, and C. G. Van de Walle, Unexpectedly strong auger recombination in halide perovskites, Advanced Energy Materials 8, 1801027 (2018)

[43] A. Surrente, A. A. Mitioglu, K. Galkowski, L. Klopotowski, W. Tabis, B. Vignolle, D. K. Maude, and P. Plochocka, Onset of exciton-exciton annihilation in singlelayer black phosphorus, Physical Review B 94, 075425 (2016).

[44] V. Pareek, J. Madeo, and K. M. Dani, Ultrafast control of the dimensionality of exciton-exciton annihilation in atomically thin black phosphorus, Physical Review Letters 124, 057403 (2020)

[45] P. Rivera, J. R. Schaibley, A. M. Jones, J. S. Ross, S. Wu, G. Aivazian, P. Klement, K. Seyler, G. Clark, N. J. Ghimire, J. Yan, D. G. Mandrus, W. Yao, and X. Xu, Observation of long-lived interlayer excitons in monolayer MoSe 2 -WSe 2 heterostructures, Nature Communications 6, 1 (2015).

[46] K. Tran, G. Moody, F. Wu, X. Lu, J. Choi, K. Kim, A. Rai, D. A. Sanchez, J. Quan, A. Singh, J. Embley, A. Zepeda, M. Campbell, T. Autry, T. Taniguchi, K. Watanabe, N. Lu, S. K. Banerjee, K. L. Silverman, S. Kim, E. Tutuc, L. Yang, A. H. MacDonald, and X. Li, Evidence for moiré excitons in van der waals heterostructures, Nature 567, 71 (2019)

[47] P. Giannozzi, S. Baroni, N. Bonini, M. Calandra, R. Car, C. Cavazzoni, D. Ceresoli, G. L. Chiarotti, M. Cococcioni, I. Dabo, A. D. Corso, S. d. Gironcoli, S. Fabris, G. Fratesi, R. Gebauer, U. Gerstmann, C. Gougoussis, A. Kokalj, M. Lazzeri, L. Martin-Samos, N. Marzari, F. Mauri, R. Mazzarello, S. Paolini, A. Pasquarello, L. Paulatto, C. Sbraccia, S. Scandolo, G. Sclauzero, A. P. Seitsonen, A. Smogunov, P. Umari, and R. M. Wentzcovitch, QUANTUM ESPRESSO: a modular and opensource software project for quantum simulations of materials, Journal of Physics: Condensed Matter 21, 395502 (2009)

[48] P. Giannozzi, O. Andreussi, T. Brumme, O. Bunau, M. B. Nardelli, M. Calandra, R. Car, C. Cavazzoni, D. Ceresoli, M. Cococcioni, N. Colonna, I. Carnimeo, A. D. Corso, S. d. Gironcoli, P. Delugas, R. A. DiStasio, A. Ferretti, A. Floris, G. Fratesi, G. Fugallo, R. Gebauer, U. Gerstmann, F. Giustino, T. Gorni, J. Jia, M. Kawamura, H.-Y. Ko, A. Kokalj, E. Küçükbenli, M. Lazzeri, M. Marsili, N. Marzari, F. Mauri, N. L. Nguyen, H.V. Nguyen, A. Otero-de-la Roza, L. Paulatto, S. Poncé, D. Rocca, R. Sabatini, B. Santra, M. Schlipf, A. P. Seitsonen, A. Smogunov, I. Timrov, T. Thonhauser, P. Umari, N. Vast, X. Wu, and S. Baroni, Advanced capabilities for materials modelling with quantum ESPRESSO, Journal of Physics: Condensed Matter 29, 465901 (2017)

[49] J. P. Perdew, K. Burke, and M. Ernzerhof, Generalized gradient approximation made simple, Physical Review Letters 77, 3865 (1996)

[50] J. P. Perdew, K. Burke, and M. Ernzerhof, Generalized gradient approximation made simple [Phys. rev. lett. 77, 3865 (1996)], Physical Review Letters 78, 1396 (1997).

[51] A. Dal Corso, Pseudopotentials periodic table: From h to pu, Computational Materials Science 95, 337 (2014).

[52] T. Björkman, Testing several recent van der waals density functionals for layered structures, The Journal of Chemical Physics 141, 074708 (2014).

[53] A. Molina-Sánchez, K. Hummer, and L. Wirtz, Vibrational and optical properties of MoS2: from monolayer to bulk, Surface Science Reports 70, 554 (2015)

[54] K. Nakamura, Y. Yoshimoto, Y. Nomura, T. Tadano, M. Kawamura, T. Kosugi, K. Yoshimi, T. Misawa, and Y. Motoyama, RESPACK: an ab initio tool for derivation of effective low-energy model of material, Computer Physics Communications 261, 107781 (2021).

[55] G.-B. Liu, W.-Y. Shan, Y. Yao, W. Yao, and D. Xiao, Three-band tight-binding model for monolayers of groupVIB transition metal dichalcogenides, Physical Review B 
88, $085433(2013)$.

[56] R. Resta, Thomas-fermi dielectric screening in semiconductors, Physical Review B 16, 2717 (1977)

[57] I. Kylänpää and H.-P. Komsa, Binding energies of exciton complexes in transition metal dichalcogenide monolayers and effect of dielectric environment, Physical Review B 92, 205418 (2015).

\section{APPENDIX}

A. Density functional theory calculations, spin-orbit coupling and Coulomb matrix elements

Density functional theory (DFT) calculations for freestanding monolayer $\mathrm{MoS}_{2}$ are carried out using QUANTUM ESPRESSO V.6.6 [47, 48. We apply the generalized gradient approximation (GGA) by Perdew, Burke, and Ernzerhof (PBE) [49, 50] and use projectoraugmented wave (PAW) pseudopotentials from the PSLibrary 51 at a plane-wave cutoff of $50 \mathrm{Ry}$. Uniform meshes (including the $\Gamma$-point) with $18 \times 18 \times 1 \mathrm{k}$-points are combined with a Fermi-Dirac smearing of $5 \mathrm{mRy}$. Using a fixed lattice constant of $a=3.16 \AA[52$, 553] and a fixed cell height of $45 \AA$, forces are minimized below $10^{-3} \mathrm{eV} / \AA$.

We use RESPACK 54 to construct a lattice Hamiltonian $H_{0}(\mathbf{k})$ in an 11-dimensional localized basis of Wannier orbitals $\left(\mathrm{d}_{z^{2}}, \mathrm{~d}_{x z}, \mathrm{~d}_{y z}, \mathrm{~d}_{x^{2}-y^{2}}\right.$ and $\mathrm{d}_{x y}$ for Mo, $\mathrm{p}_{x}, \mathrm{p}_{y}$ and $\mathrm{p}_{z}$ for $\mathrm{S}$ ) from the DFT results. We also calculate the dielectric function as well as bare and screened Coulomb matrix elements in the localized basis. For the polarization function, a cutoff energy of 5 Ry, 96 bands as well as 70 frequency points on a logarithmic grid are taken into account.

Spin-orbit interaction is included using an on-site $\boldsymbol{L} \cdot \boldsymbol{S}$ coupling Hamiltonian along the lines of [55], which is added to the non-relativistic Wannier Hamiltonian:

$$
H(\mathbf{k})=I_{2} \otimes H_{0}(\mathbf{k})+H_{\mathrm{SOC}} .
$$

Here, $I_{2}$ is the $2 \times 2$ identity matrix in the Hilbert space spanned by eigenstates $|\uparrow\rangle$ and $|\downarrow\rangle$ of the spin z component (perpendicular to the monolayer). By treating spatial degrees of freedom and spin separately, we reduce the size of the Wannier Hamiltonian and thereby the Coulomb matrix. We assume that the Coulomb matrix is spin-independent. Diagonalization of $H(\mathbf{k})$ yields the band structure $\varepsilon_{\mathbf{k}}^{\lambda}$ and the Bloch states $\left|\psi_{\mathbf{k}}^{\lambda}\right\rangle=$ $\sum_{\alpha} c_{\alpha, \mathbf{k}}^{\lambda}|\mathbf{k}, \alpha\rangle$, where the coefficients $c_{\alpha, \mathbf{k}}^{\lambda}$ describe the momentum-dependent contribution of the orbital $\alpha$ to the Bloch band $\lambda$. The Bloch sums $|\mathbf{k}, \alpha\rangle$ are connected to the localized basis via $|\mathbf{k}, \alpha\rangle=\frac{1}{\sqrt{N}} \sum_{\mathbf{R}} e^{i \mathbf{k} \cdot \mathbf{R}}|\mathbf{R}, \alpha\rangle$ with the number of unit cells $N$ and lattice vectors $\mathbf{R}$. The SOC-Hamiltonian is given by

$$
H_{\mathrm{SOC}}=\frac{1}{\hbar^{2}} \tilde{\boldsymbol{L}} \cdot \boldsymbol{S}=\frac{1}{2 \hbar} \tilde{\boldsymbol{L}} \cdot \boldsymbol{\sigma}
$$

with the Pauli matrices $\boldsymbol{\sigma}=\left(\sigma_{x}, \sigma_{y}, \sigma_{z}\right)$ and the modified angular momentum operator

$$
\tilde{\boldsymbol{L}}=\left(\begin{array}{ccc}
\lambda_{\mathrm{Mo}} \boldsymbol{L}_{l=2} & 0 & 0 \\
0 & \lambda_{\mathrm{S}} \boldsymbol{L}_{l=1} & 0 \\
0 & 0 & \lambda_{\mathrm{S}} \boldsymbol{L}_{l=1}
\end{array}\right)
$$

that contains intra-atomic coupling parameters $\lambda_{\mathrm{Mo}}$ for the $(l=2)$-subspace $\left(\mathrm{d}_{z^{2}}, \mathrm{~d}_{x z}, \mathrm{~d}_{y z}, \mathrm{~d}_{x^{2}-y^{2}}\right.$ and $\left.\mathrm{d}_{x y}\right)$ and 
$\lambda_{\mathrm{S}}$ for the $(l=1)$-subspace $\left(\mathrm{p}_{x}, \mathrm{p}_{y}\right.$ and $\left.\mathrm{p}_{z}\right)$. In the given basis, the angular momentum algebra for spherical harmonics yields:

$$
\begin{aligned}
L_{x, l=1} & =\left(\begin{array}{ccc}
0 & 0 & 0 \\
0 & 0 & -i \hbar \\
0 & i \hbar & 0
\end{array}\right), L_{y, l=1}=\left(\begin{array}{ccc}
0 & 0 & i \hbar \\
0 & 0 & 0 \\
-i \hbar & 0 & 0
\end{array}\right), \\
L_{z, l=1} & =\left(\begin{array}{ccc}
0 & -i \hbar & 0 \\
i \hbar & 0 & 0 \\
0 & 0 & 0
\end{array}\right)
\end{aligned}
$$

and

$$
\begin{aligned}
L_{x, l=2} & =\left(\begin{array}{ccccc}
0 & 0 & \sqrt{3} i \hbar & 0 & 0 \\
0 & 0 & 0 & 0 & i \hbar \\
-\sqrt{3} i \hbar & 0 & 0 & -i \hbar & 0 \\
0 & 0 & i \hbar & 0 & 0 \\
0 & -i \hbar & 0 & 0 & 0
\end{array}\right), \\
L_{y, l=2} & =\left(\begin{array}{ccccc}
0 & -\sqrt{3} i \hbar & 0 & 0 & 0 \\
\sqrt{3} i \hbar & 0 & 0 & -i \hbar & 0 \\
0 & 0 & 0 & 0 & -i \hbar \\
0 & i \hbar & 0 & 0 & 0 \\
0 & 0 & i \hbar & 0 & 0
\end{array}\right), \\
L_{z, l=2} & =\left(\begin{array}{ccccc}
0 & 0 & 0 & 0 & 0 \\
0 & 0 & -i \hbar & 0 & 0 \\
0 & i \hbar & 0 & 0 & 0 \\
0 & 0 & 0 & 0 & -2 i \hbar \\
0 & 0 & 0 & 2 i \hbar & 0
\end{array}\right)
\end{aligned}
$$

We choose the coupling constants $\lambda_{\mathrm{Mo}}=90 \mathrm{meV}$ and $\lambda_{\mathrm{S}}=20 \mathrm{meV}$ to reproduce the spin-orbit splittings at the K-point as obtained from DFT calculations including spin-orbit coupling using fully relativistic pseudopotentials. The valence-band and conduction-band splittings are $146 \mathrm{meV}$ and $3 \mathrm{meV}$, respectively, with a likespin ground state. Fig. S1] shows the excellent agreement between band structures directly from fully relativistic DFT calculations and from diagonalization of the spinaugmented Wannier Hamiltonian (S1).

Starting from the density-density-like bare Coulomb interaction matrix elements in the Wannier basis,

$$
\begin{aligned}
U_{\alpha \beta}(\mathbf{q}) & =\sum_{\mathbf{R}} e^{i \mathbf{q} \cdot \mathbf{R}} U_{\alpha \beta \beta \alpha}(\mathbf{R}) \\
& =\sum_{\mathbf{R}} e^{i \mathbf{q} \cdot \mathbf{R}}\left\langle\mathbf{0}, \alpha\left|\left\langle\mathbf{R}, \beta\left|U\left(\mathbf{r}, \mathbf{r}^{\prime}\right)\right| \mathbf{R}, \beta\right\rangle\right| \mathbf{0}, \alpha\right\rangle,
\end{aligned}
$$

and the corresponding (statically) screened matrix elements $V_{\alpha \beta}(\mathbf{q})$, we obtain an analytic description of Coulomb interaction in freestanding monolayer TMDs that can be extended to include screening from a dielectric environment. To this end, we diagonalize the bare Coulomb matrix $\boldsymbol{U}(\mathbf{q})$ to obtain eigenvalues $U_{i}(\mathbf{q})$ and eigenvectors $\boldsymbol{e}_{i}(\mathbf{q})$. Since the momentum-dependence of the eigenvectors is weak, we use their long-wavelength limit in the following. The leading four eigenvalues are shown in Fig. S2, All further values are of similar size as

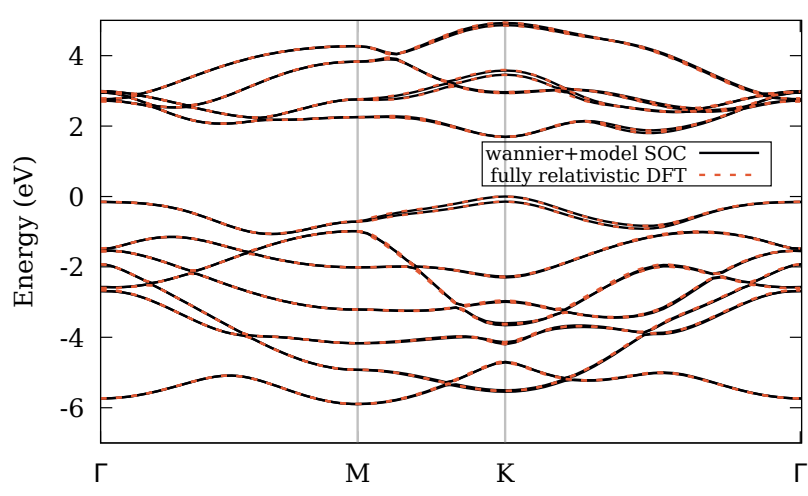

FIG. S1. Band structure of freestanding monolayer $\mathrm{MoS}_{2}$ as obtained from a fully relativistic DFT calculation (dashed red lines) compared to a non-relativistic Wannier construction augmented by a $\boldsymbol{L} \cdot \boldsymbol{S}$-Hamiltonian (solid black lines).

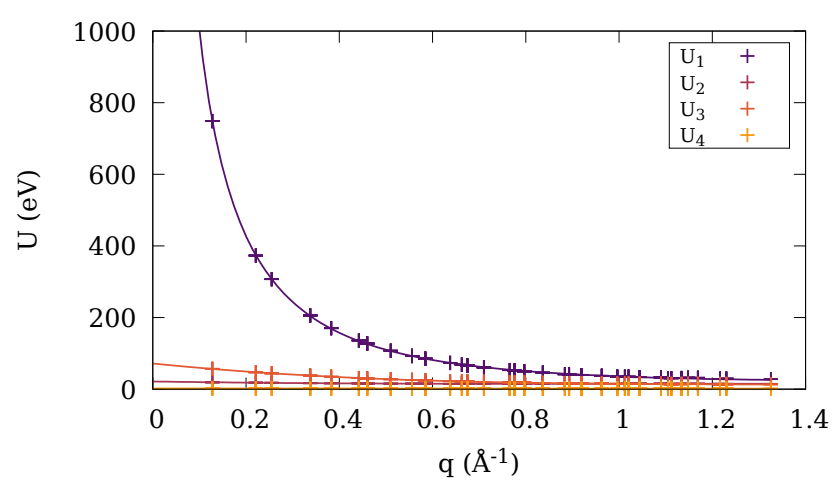

FIG. S2. Leading four eigenvalues of the bare Coulomb matrix for freestanding monolayer $\mathrm{MoS}_{2}$ (symbols) and analytic fit functions (solid lines) as discussed in the text.

$U_{4}(\mathbf{q})$. For the analytic description of the leading eigenvalue, we use

$$
U_{1}(q)=\frac{n_{\mathrm{orb}} e^{2}}{2 \varepsilon_{0} A_{\mathrm{UC}}} \frac{1}{q\left(1+\gamma q+\delta q^{2}+\eta q^{3}\right)},
$$

where the area of the hexagonal unit cell $A_{\mathrm{UC}}=\frac{\sqrt{3}}{2} a^{2}$ and the number of orbitals $n_{\text {orb }}=11$ ensure the proper normalization of Coulomb matrix elements. The eigenvalues $U_{2}(q)$ through $U_{11}(q)$ are fitted by third-order polynomials. The matrix elements of the screened interaction $\boldsymbol{V}(q)$ in the eigenbasis of the bare interaction are then obtained via

$$
V_{i}(q)=\varepsilon_{i}^{-1}(q) U_{i}(q),
$$

where the dielectric matrix $\varepsilon(q)$ accounts for both the material-specific internal polarizability and the screening by the environment. First, we introduce an analytic description for the freestanding monolayer dielectric function, i.e. in the absence of external screening. While the 


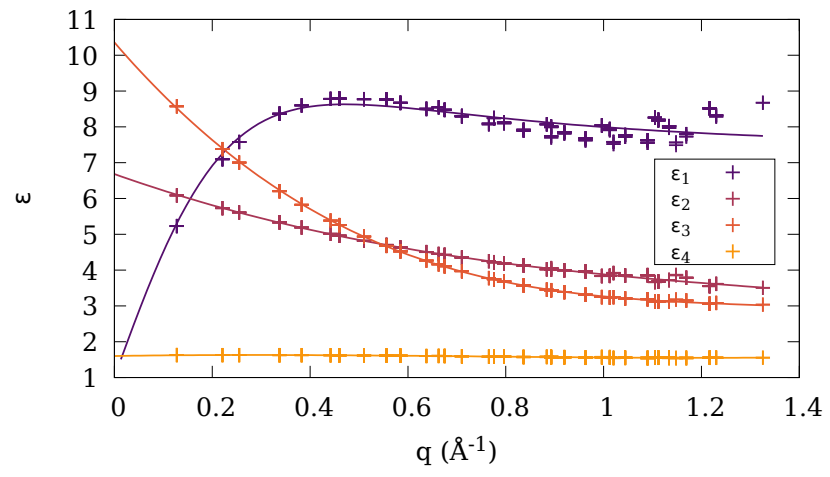

FIG. S3. Leading four eigenvalues of the dielectric matrix for freestanding monolayer $\mathrm{MoS}_{2}$ (symbols) and analytic fit functions (solid lines) as discussed in the text.

eigenvalues $\varepsilon_{2}(q)$ through $\varepsilon_{11}(q)$ are again well described by third-order polynomials, the leading eigenvalue is expressed by

$$
\varepsilon_{1}(q)=\varepsilon_{\infty}(q) \frac{1-\beta^{2} e^{-2 q d}}{1+2 \beta e^{-q d}+\beta^{2} e^{-2 q d}},
$$

with $\beta=\frac{\varepsilon_{\infty}(q)-1}{\varepsilon_{\infty}(q)+1}[32$ and the bulk dielectric constant given by a modified Resta model [56]

$$
\varepsilon_{\infty}(q)=\frac{a+q^{2}}{\frac{a \sin (q c)}{q b c}+q^{2}}+e .
$$

As layer thickness, we use the layer separation in bulk $d=0.62 \mathrm{~nm}$ [57. The leading four eigenvalues are shown in Fig. S3. As soon as all fitting parameters are obtained, environmental screening can be taken into account according to the Wannier function continuum electrostatics approach 32 that combines a macroscopic electrostatic model for the screening by the dielectric environment with a localized description of Coulomb interaction. The leading eigenvalue $\varepsilon_{1}(q)$, which is most sensitive to macroscopic screening, is modified by replacing the dielectric function of a freestanding monolayer with that of an arbitrary vertical heterostructure. The latter is obtained by solving Poisson's equation for a test charge in a slab with thickness $d$ and dielectric function $\varepsilon_{\infty}(q)$ embedded in a z-dependent dielectric profile 34. After calculating the eigenvalues $V_{i}(q)$, the Coulomb matrix in the Wannier basis is obtained by using the eigenvectors $\boldsymbol{e}_{i}(\mathbf{q})$. We finally compute screened Coulomb matrix elements in the Bloch-state representation by a unitary transformation using the coefficients $c_{\alpha, \mathbf{k}}^{\lambda}$ :

$$
V_{\mathbf{k}_{1}, \mathbf{k}_{2}, \mathbf{k}_{3}, \mathbf{k}_{4}}^{\lambda, \nu, \nu^{\prime}, \lambda^{\prime}}=\frac{1}{N} \sum_{\alpha, \beta}\left(c_{\alpha, \mathbf{k}_{1}}^{\lambda}\right)^{*}\left(c_{\beta, \mathbf{k}_{2}}^{\nu}\right)^{*} V_{\left|\mathbf{k}_{1}-\mathbf{k}_{4}\right|}^{\alpha \beta} c_{\beta, \mathbf{k}_{3}}^{\nu^{\prime}} c_{\alpha, \mathbf{k}_{4}}^{\lambda^{\prime}},
$$

where $\mathbf{k}_{4}=\mathbf{k}_{1}+\mathbf{k}_{2}-\mathbf{k}_{3}+\mathbf{G}$ due to momentum conservation and the factor $1 / N$ stems from the normalization of Wannier functions. Note that $V_{\mathbf{q}}^{\alpha \beta}$ is periodic with respect to reciprocal lattice vectors $\mathbf{G}$. In the following, we pull $1 /\left(N A_{\mathrm{UC}}\right)=1 / \mathcal{A}$ out of the matrix elements.

For a TMD monolayer, the spin z component is a good quantum number due to the reflection symmetry by the $\mathrm{x}-\mathrm{y}$ plane [21, 55]. Every Bloch band can be assigned to $|\uparrow\rangle$ or $|\downarrow\rangle$. Since this symmetry can be slightly violated due to inaccuracies of our model, we enforce it by assigning a definite spin to each band according to the dominant contribution given by the coefficients $c_{\alpha, \mathbf{k}}^{\lambda}$. Furthermore, we make use of the fact that Coulomb interaction is spin-conserving, so that we can set Coulomb matrix elements $V_{\mathbf{k}_{1} \mathbf{k}_{2} \mathbf{k}_{3} \mathbf{k}_{4}, \nu, \nu^{\prime}}$ to zero if $\lambda$ and $\lambda^{\prime}$ or $\nu$ and $\nu^{\prime}$ belong to different spins.

\section{B. Derivation of microscopic EEA scattering rates}

We start from a Hamiltonian for Bloch electrons interacting via a statically screened Coulomb potential $V$ :

$$
\begin{aligned}
H & =H_{0}+H_{\text {Coul }} \\
& =\sum_{\mathbf{k}, c} \varepsilon_{\mathbf{k}}^{c} a_{\mathbf{k}, c}^{\dagger} a_{\mathbf{k}, c}+\sum_{\mathbf{k}, v} \varepsilon_{\mathbf{k}}^{v} a_{\mathbf{k}, v}^{\dagger} a_{\mathbf{k}, v} \\
& +\frac{1}{2 \mathcal{A}} \sum_{\substack{\mathbf{k}, \mathbf{k}^{\prime}, \mathbf{q} \\
\lambda, \nu, \nu^{\prime}, \lambda^{\prime}}} V_{\mathbf{k}, \mathbf{k}^{\prime}, \mathbf{k}^{\prime}+\mathbf{q}, \mathbf{k}-\mathbf{q}}^{\lambda a_{\mathbf{k}, \lambda}^{\dagger}} a_{\mathbf{k}^{\prime}, \nu}^{\dagger} a_{\mathbf{k}^{\prime}+\mathbf{q}, \nu^{\prime}} a_{\mathbf{k}-\mathbf{q}, \lambda^{\prime}},
\end{aligned}
$$

where $a_{\mathbf{k}, c / v}^{\dagger}$ and $a_{\mathbf{k}, c / v}$ denote creation and annihilation operators, respectively, for a carrier with momentum $\mathbf{k}$ in a conduction/valence band. The single-particle band structure is given by $\varepsilon_{\mathbf{k}}^{\lambda}$. To discuss quantities in an exciton picture, we introduce two-particle operators

$$
\begin{aligned}
X_{\alpha, \mathbf{q}}^{\dagger} & =\sum_{\mathbf{k}, v, c} \phi_{\alpha, \mathbf{k}}^{v c}(\mathbf{q}) a_{\mathbf{k}-\mathbf{q}, \mathrm{c}}^{\dagger} a_{\mathbf{k}, \mathrm{v}}, \\
a_{\mathbf{k}-\mathbf{q}, \mathrm{c}}^{\dagger} a_{\mathbf{k}, \mathrm{v}} & =\sum_{\alpha}\left(\phi_{\alpha, \mathbf{k}}^{v c}(\mathbf{q})\right)^{*} X_{\alpha, \mathbf{q}}^{\dagger} .
\end{aligned}
$$

The wave functions $\phi_{\alpha, \mathbf{k}}^{v c}(\mathbf{q})$ are solutions of the BetheSalpeter equation (BSE) in the absence of photoexcited carriers

$$
\begin{gathered}
\left(\varepsilon_{\mathbf{k}-\mathbf{q}}^{\mathrm{c}}-\varepsilon_{\mathbf{k}}^{\mathrm{v}}-E_{\alpha, \mathbf{q}}\right) \phi_{\alpha, \mathbf{k}}^{v c}(\mathbf{q}) \\
-\frac{1}{\mathcal{A}} \sum_{\mathbf{k}^{\prime}, v^{\prime}, c^{\prime}} V_{\mathbf{k}-\mathbf{q}, \mathbf{k}^{\prime}, \mathbf{k}, \mathbf{k}^{\prime}-\mathbf{q}}^{c, \phi^{\prime}, \phi_{\alpha, \mathbf{k}^{\prime}}^{v^{\prime} c^{\prime}}(\mathbf{q})=0,}
\end{gathered}
$$

with two-particle eigenenergies $E_{\alpha, \mathbf{q}}$ and the crystal area $\mathcal{A}$. The total momentum is denoted by $\mathbf{q}$, while $\alpha$ is the quantum number that belongs to the relative motion of electron and hole. The wave functions fulfill orthonormality and completeness relations:

$$
\begin{aligned}
& \sum_{\mathbf{k}, v, c}\left(\phi_{\alpha, \mathbf{k}}^{v, c}(\mathbf{q})\right)^{*} \phi_{\alpha^{\prime}, \mathbf{k}}^{v, c}(\mathbf{q})=\delta_{\alpha, \alpha^{\prime}}, \\
& \sum_{\alpha} \phi_{\alpha, \mathbf{k}}^{v, c}(\mathbf{q})\left(\phi_{\alpha, \mathbf{k}^{\prime}}^{v^{\prime}, c^{\prime}}(\mathbf{q})\right)^{*}=\delta_{\mathbf{k}, \mathbf{k}^{\prime}} \delta_{v, v^{\prime}} \delta_{c, c^{\prime}}
\end{aligned}
$$


Auger-like EEA emerges as a higher-order carrier-carrier interaction process within the dynamics of microscopic exciton populations, which are described by two-particle correlation functions (doublets) $n_{\alpha, \mathbf{q}}=\Delta\left\langle X_{\alpha, \mathbf{q}}^{\dagger} X_{\alpha, \mathbf{q}}\right\rangle$ [25. Correlation functions are defined recursively as the difference between an expectation value and all of its possible factorizations into smaller correlation functions, i.e. with less operators 25]. For example, a general doublet is given by

$$
\begin{aligned}
& \Delta\left\langle a_{\mathbf{k}-\mathbf{q}, 1}^{\dagger} a_{\mathbf{k}^{\prime}+\mathbf{q}, 2}^{\dagger} a_{\mathbf{k}^{\prime}, 3} a_{\mathbf{k}, 4}\right\rangle \\
& =\left\langle a_{\mathbf{k}-\mathbf{q}, 1}^{\dagger} a_{\mathbf{k}^{\prime}+\mathbf{q}, 2}^{\dagger} a_{\mathbf{k}^{\prime}, 3} a_{\mathbf{k}, 4}\right\rangle \\
& -\Delta\left\langle a_{\mathbf{k}-\mathbf{q}, 1}^{\dagger} a_{\mathbf{k}, 4}\right\rangle \Delta\left\langle a_{\mathbf{k}^{\prime}+\mathbf{q}, 2}^{\dagger} a_{\mathbf{k}^{\prime}, 3}\right\rangle \delta_{\mathbf{q}, \mathbf{0}} \\
& +\Delta\left\langle a_{\mathbf{k}-\mathbf{q}, 1}^{\dagger} a_{\mathbf{k}^{\prime}, 3}\right\rangle \Delta\left\langle a_{\mathbf{k}^{\prime}+\mathbf{q}, 2}^{\dagger} a_{\mathbf{k}, 4}\right\rangle \delta_{\mathbf{q}, \mathbf{k}-\mathbf{k}^{\prime}}
\end{aligned}
$$

Note that translational invariance of the crystal enforces momentum conservation within any expectation value and that transposition of fermionic operators yields a minus sign. The dynamics of operators is governed by the Heisenberg EOM:

$$
i \hbar \frac{d}{d t} \hat{A}=[\hat{A}, H]
$$

We apply the so-called cluster expansion technique [25] to formulate the dynamical equations in terms of correlation functions instead of expectation values. To this end, we make use of the identity

$$
[\hat{A} \hat{B}, \hat{C}]=\hat{A}[\hat{B}, \hat{C}]_{+}-[\hat{A}, \hat{C}]_{+} \hat{B}
$$

where $[\cdot, \cdot]_{+}$denotes the anti-commutator.

The $n_{\alpha, \mathbf{q}}$ are transformed to the Bloch representation using the expansion of two-particle operators (S13):

$$
\begin{aligned}
& n_{\alpha, \mathbf{q}}=\Delta\left\langle X_{\alpha, \mathbf{q}}^{\dagger} X_{\alpha, \mathbf{q}}\right\rangle \\
& =\sum_{\substack{\mathbf{k}, v, c \\
\mathbf{k}^{\prime}, v^{\prime}, c^{\prime}}} \phi_{\alpha, \mathbf{k}^{\prime}}^{v^{\prime}, c^{\prime}}(\mathbf{q})\left(\phi_{\alpha, \mathbf{k}}^{v, c}(\mathbf{q})\right)^{*} \Delta\left\langle a_{\mathbf{k}^{\prime}-\mathbf{q}, c^{\prime}}^{\dagger} a_{\mathbf{k}^{\prime}, v^{\prime}} a_{\mathbf{k}, v}^{\dagger} a_{\mathbf{k}-\mathbf{q}, c}\right\rangle \\
& =\sum_{\substack{\mathbf{k}, v, c \\
\mathbf{k}^{\prime}, v^{\prime}, c^{\prime}}} \phi_{\alpha, \mathbf{k}^{\prime}}^{v^{\prime}, c^{\prime}}(\mathbf{q})\left(\phi_{\alpha, \mathbf{k}}^{v, c}(\mathbf{q})\right)^{*} \Delta\left\langle a_{\mathbf{k}, v}^{\dagger} a_{\mathbf{k}^{\prime}-\mathbf{q}, c^{\prime}}^{\dagger} a_{\mathbf{k}^{\prime}, v^{\prime}} a_{\mathbf{k}-\mathbf{q}, c}\right\rangle \\
& =\sum_{\substack{\mathbf{k}, v, c \\
\mathbf{k}^{\prime}, v^{\prime}, c^{\prime}}} \phi_{\alpha, \mathbf{k}^{\prime}+\mathbf{q}}^{v^{\prime}, c^{\prime}}(\mathbf{q})\left(\phi_{\alpha, \mathbf{k}}^{v, c}(\mathbf{q})\right)^{*} \Delta\left\langle a_{\mathbf{k}, v}^{\dagger} a_{\mathbf{k}^{\prime}, c^{\prime}}^{\dagger} a_{\mathbf{k}^{\prime}+\mathbf{q}, v^{\prime}} a_{\mathbf{k}-\mathbf{q}, c}\right\rangle .
\end{aligned}
$$

Within a correlation function, transposition of two fermionic operators yields a minus sign [25]. The time derivative of the carrier-carrier correlation in the Bloch picture can be evaluated by commutating with the Hamiltonian (S12). Since we are interested in the dynamics due to EEA, we retain only coupling terms to three-particle correlation functions (triplets). The other terms are discussed at length in Ref. 25]. This yields:

$$
\begin{aligned}
& i \hbar \frac{d}{d t} n_{\alpha, \mathbf{q}}=\sum_{\mathbf{k}^{\prime}, v^{\prime}, c^{\prime}} \phi_{\alpha, \mathbf{k}^{\prime}+\mathbf{q}}^{v^{\prime}, c^{\prime}}(\mathbf{q}) \sum_{\mathbf{k}, v, c}\left(\phi_{\alpha, \mathbf{k}}^{v, c}(\mathbf{q})\right)^{*} \frac{1}{\mathcal{A}} \sum_{\mathbf{l}, \mathbf{l}^{\prime}, 1,2,3} \times \\
& \left\{V_{\mathbf{k}-\mathbf{q}, \mathbf{l}, \mathbf{l}+\mathbf{l}^{\prime}, \mathbf{k}-\mathbf{q}-\mathbf{l}^{\prime}}^{c, 1} \Delta\left\langle a_{\mathbf{k}, v}^{\dagger} a_{\mathbf{k}^{\prime}, c^{\prime}}^{\dagger} a_{\mathbf{l}, 1}^{\dagger} a_{\mathbf{l}+\mathbf{l}^{\prime}, 2} a_{\mathbf{k}^{\prime}+\mathbf{q}, v^{\prime}} a_{\mathbf{k}-\mathbf{q}-\mathbf{l}^{\prime}, 3}\right\rangle\right. \\
& +V_{\mathbf{k}^{\prime}+\mathbf{q}, \mathbf{l}, \mathbf{l}+\mathbf{l}^{\prime}, \mathbf{k}^{\prime}+\mathbf{q}-\mathbf{l}^{\prime}}^{v^{\prime}, 1,2,3}\left\langle a_{\mathbf{k}, v}^{\dagger} a_{\mathbf{k}^{\prime}, c^{\prime}}^{\dagger} a_{\mathbf{l}, 1}^{\dagger} a_{\mathbf{l}+\mathbf{l}^{\prime}, 2} a_{\mathbf{k}^{\prime}+\mathbf{q}-\mathbf{l}^{\prime}, 3} a_{\mathbf{k}-\mathbf{q}, c}\right\rangle \\
& -\left(V_{\mathbf{k}^{\prime}, \mathbf{l}, \mathbf{l}+\mathbf{l}^{\prime}, \mathbf{k}^{\prime}-\mathbf{l}^{\prime}}^{c^{\prime}} \Delta\left\langle a_{\mathbf{k}-\mathbf{q}, c^{\prime}}^{\dagger} a_{\mathbf{k}^{\prime}+\mathbf{q}, v^{\prime}}^{\dagger} a_{\mathbf{l}, 1}^{\dagger} a_{\mathbf{l}+\mathbf{l}^{\prime}, 2} a_{\mathbf{k}^{\prime}-\mathbf{l}^{\prime}, 3} a_{\mathbf{k}, v}\right\rangle\right)^{*} \\
& \left.-\left(V_{\mathbf{k}, \mathbf{l}, \mathbf{l}+\mathbf{l}^{\prime}, \mathbf{k}-\mathbf{l}^{\prime}}^{v, 1,3}\left\langle a_{\mathbf{k}-\mathbf{q}, c}^{\dagger} a_{\mathbf{k}^{\prime}+\mathbf{q}, v^{\prime}}^{\dagger} a_{\mathbf{l}, 1}^{\dagger} a_{\mathbf{l}+\mathbf{l}^{\prime}, 2} a_{\mathbf{k}^{\prime}, c^{\prime}} a_{\mathbf{k}-\mathbf{l}^{\prime}, 3}\right\rangle\right)^{*}\right\} .
\end{aligned}
$$

We keep all band indices 1,2,3 that allow for a pairing of conduction and valence band operators into two-particle operators, which yields three possible combinations for each of the terms in Eq. S20. After re-introducing twoparticle operators and using the orthonormality relations for wave functions, the 12 resulting terms can by rearranged as follows:

$$
\begin{aligned}
& i \hbar \frac{d}{d t} n_{\alpha, \mathbf{q}}=\frac{1}{\mathcal{A}} \sum_{\mathbf{l}^{\prime}} \times \\
& 2 i \operatorname{Im}\left\{\sum_{\beta \delta}\left(\hat{V}_{\alpha, \beta, \delta, \mathbf{q}, \mathbf{l}^{\prime}}^{(1), D}\right)^{*} \Delta\left\langle X_{\alpha, \mathbf{q}}^{\dagger} X_{\beta, \mathbf{q}+\mathbf{l}^{\prime}} X_{\delta,-\mathbf{l}^{\prime}}\right\rangle\right. \\
+ & \sum_{\beta \delta}\left(\hat{V}_{\beta, \alpha, \delta, \mathbf{q}+\mathbf{l}^{\prime},-\mathbf{l}^{\prime}}^{(1), D}-\hat{V}_{\beta, \alpha, \delta, \mathbf{q}+\mathbf{l}^{\prime},-\mathbf{l}^{\prime}}^{(1), X}\left(\Delta\left\langle X_{\beta, \mathbf{q}+\mathbf{l}^{\prime}}^{\dagger} X_{\alpha, \mathbf{q}} X_{\delta, \mathbf{l}^{\prime}}\right\rangle\right)^{*}\right. \\
+ & \sum_{\beta \delta}\left(\left(\hat{V}_{\beta, \alpha, \delta, \mathbf{q}, \mathbf{l}^{\prime}}^{(2), D} \hat{V}_{\beta, \alpha, \delta, \mathbf{q}, \mathbf{l}^{\prime}}^{(2), X}\right)\right)^{*} \Delta\left\langle X_{\beta, \mathbf{q}-\mathbf{l}^{\prime}}^{\dagger} X_{\alpha, \mathbf{q}^{\prime}} X_{\delta,-\mathbf{l}^{\prime}}\right\rangle \\
+ & \left.\sum_{\beta \delta} \hat{V}_{\alpha, \beta, \delta, \mathbf{q}-\mathbf{l}^{\prime},-\mathbf{l}^{\prime}}^{(2), D}\left(\Delta\left\langle X_{\alpha, \mathbf{q}}^{\dagger} X_{\beta, \mathbf{q}-\mathbf{l}^{\prime}} X_{\delta, \mathbf{l}^{\prime}}\right\rangle\right)^{*}\right\}
\end{aligned}
$$

where we have introduced effective direct (D) and exchange $(\mathrm{X})$ exciton-exciton interaction matrix elements $V_{\mathbf{q},-\mathbf{l}}^{D / X, \alpha, \beta, \delta}=V_{\alpha, \beta, \delta, \mathbf{q},-\mathbf{l}}^{(1), D / X}-V_{\alpha, \beta, \delta, \mathbf{q}-\mathbf{1},-\mathbf{1}}^{(2), D / X}$ as defined in Eq. (5), where $V^{(1)}$ and $V^{(2)}$ describe Auger-like scattering of electrons and holes, respectively.

Similar as for exciton populations, we use the Heisenberg equation to derive EOM for triplets:

$$
\begin{aligned}
& i \hbar \frac{d}{d t} \Delta\left\langle X_{\alpha, \mathbf{q}_{1}}^{\dagger} X_{\beta, \mathbf{q}_{2}} X_{\delta,-\mathbf{l}^{\prime}}\right\rangle \\
= & \sum_{\mathbf{k}, \mathbf{k}^{\prime}, \mathbf{l} v, c, v^{\prime}, c^{\prime}, v^{\prime \prime}, c^{\prime \prime}} \phi_{\alpha, \mathbf{k}}^{v, c}\left(\mathbf{q}_{1}\right)\left(\phi_{\beta, \mathbf{k}^{\prime}}^{v^{\prime}, c^{\prime}}\left(\mathbf{q}_{2}\right)\right)^{*}\left(\phi_{\delta, \mathbf{l}}^{v^{\prime \prime}, c^{\prime \prime}}\left(-\mathbf{l}^{\prime}\right)\right)^{*} \times \\
& i \hbar \frac{d}{d t} \Delta\left\langle a_{\mathbf{k}-\mathbf{q}_{1}, c}^{\dagger} a_{\mathbf{k}^{\prime}, v^{\prime}}^{\dagger} a_{\mathbf{l}, v^{\prime \prime}}^{\dagger} a_{\mathbf{l}+\mathbf{l}^{\prime}, c^{\prime \prime}} a_{\mathbf{k}^{\prime}-\mathbf{q}_{2}, c^{\prime}} a_{\mathbf{k}, v}\right\rangle
\end{aligned}
$$

with 


$$
\begin{aligned}
& i \hbar \frac{d}{d t} \Delta\left\langle a_{\mathbf{k}-\mathbf{q}_{1}, c}^{\dagger} a_{\mathbf{k}^{\prime}, v^{\prime}}^{\dagger} a_{\mathbf{1}, v^{\prime \prime}}^{\dagger} a_{\mathbf{1}+\mathbf{1}^{\prime}, c^{\prime \prime}} a_{\mathbf{k}^{\prime}-\mathbf{q}_{2}, c^{\prime}} a_{\mathbf{k}, v}\right\rangle \\
& =\left(\varepsilon_{\mathbf{k}}^{v}+\varepsilon_{\mathbf{k}^{\prime}-\mathbf{q}_{2}}^{c^{\prime}}+\varepsilon_{\mathbf{1}+\mathbf{1}^{\prime}}^{c^{\prime \prime}}-\varepsilon_{\mathbf{1}}^{v^{\prime \prime}}-\varepsilon_{\mathbf{k}^{\prime}}^{v^{\prime}}-\varepsilon_{\mathbf{k}-\mathbf{q}_{1}}^{c}\right) \Delta\left\langle a_{\mathbf{k}-\mathbf{q}_{1}, c^{\dagger}}^{\dagger} a_{\mathbf{k}^{\prime}, v^{\prime}}^{\dagger} a_{\mathbf{1}, v^{\prime \prime}}^{\dagger} a_{\mathbf{1}+\mathbf{1}^{\prime}, c^{\prime \prime}} a_{\mathbf{k}^{\prime}-\mathbf{q}_{2}, c^{\prime}} a_{\mathbf{k}, v}\right\rangle \\
& -\frac{1}{\mathcal{A}} \sum_{\mathbf{p}, \mathbf{p}^{\prime}, 1,2,3} V_{\mathbf{p}, \mathbf{p}^{\prime}, \mathbf{p}+\mathbf{p}^{\prime}-\left(\mathbf{k}-\mathbf{q}_{1}\right), \mathbf{k}-\mathbf{q}_{1}}^{1,2,3, c}\left\langle a_{\mathbf{p}, 1}^{\dagger} a_{\mathbf{p}^{\prime}, 2}^{\dagger} a_{\mathbf{p}+\mathbf{p}^{\prime}-\left(\mathbf{k}-\mathbf{q}_{1}\right), 3} a_{\mathbf{k}^{\prime}, v^{\prime}}^{\dagger} a_{\mathbf{1}, v^{\prime \prime}}^{\dagger} a_{\mathbf{1}+\mathbf{1}^{\prime}, c^{\prime \prime}} a_{\mathbf{k}^{\prime}-\mathbf{q}_{2}, c^{\prime}} a_{\mathbf{k}, v}\right\rangle \\
& -\frac{1}{\mathcal{A}} \sum_{\mathbf{p}, \mathbf{p}^{\prime}, 1,2,3} V_{\mathbf{p}, \mathbf{p}^{\prime}, \mathbf{p}+\mathbf{p}^{\prime}-\mathbf{k}^{\prime}, \mathbf{k}^{\prime}}^{1,2,3,}\left\langle a_{\mathbf{k}-\mathbf{q}_{1}, c}^{\dagger} a_{\mathbf{p}, 1}^{\dagger} a_{\mathbf{p}^{\prime}, 2}^{\dagger} a_{\mathbf{p}+\mathbf{p}^{\prime}-\mathbf{k}^{\prime}, 3} a_{\mathbf{1}, v^{\prime \prime}}^{\dagger} a_{\mathbf{1}+\mathbf{1}^{\prime}, c^{\prime \prime}} a_{\mathbf{k}^{\prime}-\mathbf{q}_{2}, c^{\prime}} a_{\mathbf{k}, v}\right\rangle \\
& -\frac{1}{\mathcal{A}} \sum_{\mathbf{p}, \mathbf{p}^{\prime}, 1,2,3} V_{\mathbf{p}, \mathbf{p}^{\prime}, \mathbf{p}+\mathbf{p}^{\prime}-\mathbf{1}, \mathbf{1}}^{1,2, v^{\prime \prime}}\left\langle a_{\mathbf{k}-\mathbf{q}_{1}, c}^{\dagger} a_{\mathbf{k}^{\prime}, v^{\prime}}^{\dagger} a_{\mathbf{p}, 1}^{\dagger} a_{\mathbf{p}^{\prime}, 2}^{\dagger} a_{\mathbf{p}+\mathbf{p}^{\prime}-\mathbf{1}, 3} a_{\mathbf{1}+\mathbf{1}^{\prime}, c^{\prime \prime}} a_{\mathbf{k}^{\prime}-\mathbf{q}_{2}, c^{\prime}} a_{\mathbf{k}, v}\right\rangle \\
& +\frac{1}{\mathcal{A}} \sum_{\mathbf{p}, \mathbf{p}^{\prime}, 1,2,3} V_{\mathbf{l}+\mathbf{1}^{\prime}, \mathbf{p}, \mathbf{p}+\mathbf{p}^{\prime}, \mathbf{1}+\mathbf{1}^{\prime}-\mathbf{p}^{\prime}}^{c^{\prime \prime}}\left\langle a_{\mathbf{k}-\mathbf{q}_{1}, c}^{\dagger} a_{\mathbf{k}^{\prime}, v^{\prime}}^{\dagger} a_{\mathbf{1}, v^{\prime \prime}}^{\dagger} a_{\mathbf{p}, 1}^{\dagger} a_{\mathbf{p}+\mathbf{p}^{\prime}, 2} a_{\mathbf{1}+\mathbf{1}^{\prime}-\mathbf{p}^{\prime}, 3} a_{\mathbf{k}^{\prime}-\mathbf{q}_{2}, c^{\prime}} a_{\mathbf{k}, v}\right\rangle \\
& +\frac{1}{\mathcal{A}} \sum_{\mathbf{p}, \mathbf{p}^{\prime}, 1,2,3} V_{\mathbf{k}^{\prime}-\mathbf{q}_{2}, \mathbf{p}, \mathbf{p}+\mathbf{p}^{\prime}, \mathbf{k}^{\prime}-\mathbf{q}_{2}-\mathbf{p}^{\prime}}^{c^{\prime}, 1,2}\left\langle a_{\mathbf{k}-\mathbf{q}_{1}, c}^{\dagger} a_{\mathbf{k}^{\prime}, v^{\prime}}^{\dagger} a_{\mathbf{1}, v^{\prime \prime}}^{\dagger} a_{\mathbf{l}+\mathbf{l}^{\prime}, c^{\prime \prime}} a_{\mathbf{p}, 1}^{\dagger} a_{\mathbf{p}+\mathbf{p}^{\prime}, 2} a_{\mathbf{k}^{\prime}-\mathbf{q}_{2}-\mathbf{p}^{\prime}, 3} a_{\mathbf{k}, v}\right\rangle \\
& +\frac{1}{\mathcal{A}} \sum_{\mathbf{p}, \mathbf{p}^{\prime}, 1,2,3} V_{\mathbf{k}, \mathbf{p}, \mathbf{p}+\mathbf{p}^{\prime}, \mathbf{k}-\mathbf{p}^{\prime}}^{v, 1,2}\left\langle a_{\mathbf{k}-\mathbf{q}_{1}, c}^{\dagger} a_{\mathbf{k}^{\prime}, v^{\prime}}^{\dagger} a_{\mathbf{1}, v^{\prime \prime}}^{\dagger} a_{\mathbf{1}+\mathbf{1}^{\prime}, c^{\prime \prime}} a_{\mathbf{k}^{\prime}-\mathbf{q}_{2}, c^{\prime}} a_{\mathbf{p}, 1}^{\dagger} a_{\mathbf{p}+\mathbf{p}^{\prime}, 2} a_{\mathbf{k}-\mathbf{p}^{\prime}, 3}\right\rangle \\
& -\left.i \hbar \frac{d}{d t} \Delta\left\langle a_{\mathbf{k}-\mathbf{q}_{1}, c}^{\dagger} a_{\mathbf{k}^{\prime}, v^{\prime}}^{\dagger} a_{\mathbf{1}, v^{\prime \prime}}^{\dagger} a_{\mathbf{1}+\mathbf{1}^{\prime}, c^{\prime \prime}} a_{\mathbf{k}^{\prime}-\mathbf{q}_{2}, c^{\prime}} a_{\mathbf{k}, v}\right\rangle\right|_{\text {factorizations }} .
\end{aligned}
$$

Here, the first term leading to oscillations with free energies stems from commutating with the Hamiltonian $H_{0}$, while the coupling to four-particle expectation values is due to the Coulomb interaction Hamiltonian $H_{\text {Coul }}$. Since we consider the time derivative of a correlation function, we have to subtract all terms that are due to factorizations into smaller correlation functions similar to Eq. (S16). The four-particle expectation values themselves can be represented by correlation functions according to the scheme

$$
\begin{aligned}
\langle 4\rangle & =\langle 1\rangle\langle 1\rangle\langle 1\rangle\langle 1\rangle+\langle 1\rangle\langle 1\rangle \Delta\langle 2\rangle+\Delta\langle 2\rangle \Delta\langle 2\rangle \\
& +\langle 1\rangle \Delta\langle 3\rangle+\Delta\langle 4\rangle .
\end{aligned}
$$

We discard four-particle correlations to truncate the hierarchy and obtain a closed set of equations. Moreover, we discard one-particle quantities $\Delta\left\langle a_{\mathbf{k}, \lambda}^{\dagger} a_{\mathbf{k}, \lambda^{\prime}}\right\rangle$ that are not band-diagonal as well as conduction-band populations $f_{\mathbf{k}}^{c}=\Delta\left\langle a_{\mathbf{k}, c}^{\dagger} a_{\mathbf{k}, c}\right\rangle$, while we approximate valenceband populations by 1 . By neglecting electron and hole populations, we assume that corrections due to the occupation of single-particle phase space by excitons is small. Among the doublets, we retain those which correspond to exciton populations of the states $\left|\alpha, \mathbf{q}_{1}\right\rangle,\left|\beta, \mathbf{q}_{2}\right\rangle$ or $\left|\delta,-\mathbf{l}^{\prime}\right\rangle$. Only for these factorizations, it will be possible to introduce effective interaction matrix elements as in Eq. (S21). We therefore assume that all further factorizations are beyond exciton-exciton scattering, e.g. by mixing exciton states. After cancellation between factorizations of the four-particle expectation values and the time derivative of the factorizations of triplets, we obtain: 


$$
\begin{aligned}
& i \hbar \frac{d}{d t} \Delta\left\langle a_{\mathbf{k}-\mathbf{q}_{1}, c}^{\dagger} a_{\mathbf{k}^{\prime}, v^{\prime}}^{\dagger} a_{\mathbf{1}, v^{\prime \prime}}^{\dagger} a_{\mathbf{1}+\mathbf{l}^{\prime}, c^{\prime \prime}} a_{\mathbf{k}^{\prime}-\mathbf{q}_{2}, c^{\prime}} a_{\mathbf{k}, v}\right\rangle \\
& =\left(\varepsilon_{\mathbf{k}}^{v}+\varepsilon_{\mathbf{k}^{\prime}-\mathbf{q}_{2}}^{c^{\prime}}+\varepsilon_{\mathbf{l}+\mathbf{l}^{\prime}}^{c^{\prime \prime}}-\varepsilon_{\mathbf{l}}^{v^{\prime \prime}}-\varepsilon_{\mathbf{k}^{\prime}}^{v^{\prime}}-\varepsilon_{\mathbf{k}-\mathbf{q}_{1}}^{c}\right) \times \\
& \Delta\left\langle a_{\mathbf{k}-\mathbf{q}_{1}, c^{\prime}}^{\dagger} a_{\mathbf{k}^{\prime}, v^{\prime}}^{\dagger} a_{\mathbf{1}, v^{\prime \prime}}^{\dagger} a_{\mathbf{1}+\mathbf{l}^{\prime}, c^{\prime \prime}} a_{\mathbf{k}^{\prime}-\mathbf{q}_{2}, c^{\prime}} a_{\mathbf{k}, v}\right\rangle \\
& +\frac{1}{\mathcal{A}} \sum_{\mathbf{1}^{\prime \prime}, v^{\prime \prime \prime}, c^{\prime \prime \prime}} V_{\mathbf{k}, \mathbf{1}^{\prime \prime}-\mathbf{q}_{1}, \mathbf{k}-\mathbf{q}_{1}, \mathbf{1}^{\prime \prime}}^{v, v^{\prime \prime \prime}} \Delta\left\langle a_{\mathbf{1}^{\prime \prime}-\mathbf{q}_{1}, c^{\prime \prime \prime}}^{\dagger} a_{\mathbf{k}^{\prime}, v^{\prime}}^{\dagger} a_{\mathbf{1}, v^{\prime \prime}}^{\dagger} a_{\mathbf{1}+\mathbf{l}^{\prime}, c^{\prime \prime}} a_{\mathbf{k}^{\prime}-\mathbf{q}_{2}, c^{\prime}} a_{\mathbf{1}^{\prime \prime}, v^{\prime \prime \prime}}\right\rangle \\
& -\frac{1}{\mathcal{A}} \sum_{\mathbf{1}^{\prime \prime}, v^{\prime \prime \prime}, c^{\prime \prime \prime}} V_{\mathbf{k}^{\prime}-\mathbf{q}_{2}, \mathbf{1}^{\prime \prime}, \mathbf{k}^{\prime}, \mathbf{1}^{\prime \prime}-\mathbf{q}_{2}}^{c^{\prime}, v^{\prime \prime \prime}, v^{\prime}, c^{\prime \prime \prime}} \Delta\left\langle a_{\mathbf{k}-\mathbf{q}_{1}, c^{\prime}}^{\dagger} a_{\mathbf{1}^{\prime \prime}, v^{\prime \prime \prime}}^{\dagger} a_{\mathbf{1}, v^{\prime \prime}}^{\dagger} a_{\mathbf{1}+\mathbf{l}^{\prime}, c^{\prime \prime}} a_{\mathbf{1}^{\prime \prime}-\mathbf{q}_{2}, c^{\prime \prime \prime}} a_{\mathbf{k}, v}\right\rangle \\
& -\frac{1}{\mathcal{A}} \sum_{\mathbf{1}^{\prime \prime}, v^{\prime \prime \prime}, c^{\prime \prime \prime}} V_{\mathbf{1}+\mathbf{1}^{\prime}, \mathbf{1}^{\prime \prime}, \mathbf{l}_{1} \mathbf{1}^{\prime \prime}+\mathbf{1}^{\prime}}^{c^{\prime \prime}, c^{\prime \prime \prime}} \Delta\left\langle a_{\mathbf{k}-\mathbf{q}_{1}, c}^{\dagger} a_{\mathbf{k}^{\prime}, v^{\prime}}^{\dagger} a_{\mathbf{1}^{\prime \prime}, v^{\prime \prime \prime}}^{\dagger} a_{\mathbf{1}^{\prime \prime}+\mathbf{1}^{\prime}, c^{\prime \prime \prime}} a_{\mathbf{k}^{\prime}-\mathbf{q}_{2}, c^{\prime}} a_{\mathbf{k}, v}\right\rangle \\
& +\frac{1}{\mathcal{A}} \sum_{\mathbf{p}, 1,2,3}\{ \\
& {\left[V_{\mathbf{p}, \mathbf{k}-\mathbf{q}_{1}-\mathbf{l}^{\prime}, \mathbf{p}-\mathbf{1}^{\prime}, \mathbf{k}-\mathbf{q}_{1}}^{1,2,3}-V_{\mathbf{k}-\mathbf{q}_{1}-\mathbf{l}^{\prime}, \mathbf{p}, \mathbf{p}-\mathbf{l}^{\prime}, \mathbf{k}-\mathbf{q}_{1}}^{2,1,3, c}\right] \Delta\left\langle a_{\mathbf{k}-\mathbf{q}_{1}-\mathbf{l}^{\prime}, 2}^{\dagger} a_{\mathbf{k}^{\prime}, v^{\prime}}^{\dagger} a_{\mathbf{k}^{\prime}-\mathbf{q}_{2}, c^{\prime}} a_{\mathbf{k}, v}\right\rangle \Delta\left\langle a_{\mathbf{p}, 1}^{\dagger} a_{\mathbf{1}, v^{\prime \prime}}^{\dagger} a_{\mathbf{1}+\mathbf{l}^{\prime}, c^{\prime \prime}} a_{\mathbf{p}-\mathbf{l}^{\prime}, 3}\right\rangle} \\
& +\left[V_{\mathbf{p}, \mathbf{k}+\mathbf{1}^{\prime}, \mathbf{p}+\mathbf{l}^{\prime}+\mathbf{q}_{1}, \mathbf{k}-\mathbf{q}_{1}}^{1,2,}-V_{\mathbf{k}+\mathbf{l}^{\prime}, \mathbf{p}, \mathbf{p}+\mathbf{l}^{\prime}+\mathbf{q}_{1}, \mathbf{k}-\mathbf{q}_{1}}^{2,1,3}\right] \Delta\left\langle a_{\mathbf{k}+\mathbf{l}^{\prime}, 2}^{\dagger} a_{\mathbf{1}, v^{\prime \prime}}^{\dagger} a_{\mathbf{l}+\mathbf{l}^{\prime}, c^{\prime \prime}} a_{\mathbf{k}, v}\right\rangle \Delta\left\langle a_{\mathbf{p}, 1}^{\dagger} a_{\mathbf{k}^{\prime}, v^{\prime}}^{\dagger} a_{\mathbf{k}^{\prime}-\mathbf{q}_{2}, c^{\prime}} a_{\mathbf{p}+\mathbf{q}_{2}, 3}\right\rangle \\
& \left.-\left[V_{\mathbf{p}, \mathbf{k}^{\prime}-\mathbf{l}^{\prime}, \mathbf{p}-\mathbf{l}^{\prime}, \mathbf{k}^{\prime}}^{1,2, v^{\prime}}-V_{\mathbf{k}^{\prime}-\mathbf{l}^{\prime}, \mathbf{p}, \mathbf{p}-\mathbf{l}^{\prime}, \mathbf{k}^{\prime}}^{2,1,3, v^{\prime}}\right] \Delta a_{\mathbf{k}^{\prime}-\mathbf{l}^{\prime}, 2}^{\dagger} a_{\mathbf{k}-\mathbf{q}_{1}, c^{\prime}}^{\dagger} a_{\mathbf{k}^{\prime}-\mathbf{q}_{2}, c^{\prime}} a_{\mathbf{k}, v}\right\rangle \Delta\left\langle a_{\mathbf{p}, 1}^{\dagger} a_{\mathbf{l}, v^{\prime \prime}}^{\dagger} a_{\mathbf{1}+\mathbf{l}^{\prime}, c^{\prime \prime}} a_{\mathbf{p}-\mathbf{l}^{\prime}, 3}\right\rangle
\end{aligned}
$$

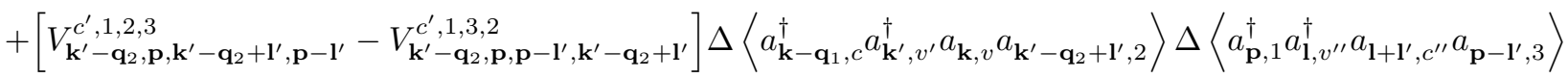

$$
\begin{aligned}
& -\left[V_{\mathbf{k}, \mathbf{p}, \mathbf{k}-\mathbf{q}_{1}-\mathbf{l}^{\prime}, \mathbf{p}+\mathbf{q}_{2}}^{v, 1,2}-V_{\mathbf{k}, \mathbf{p}, \mathbf{p}+\mathbf{q}_{2}, \mathbf{k}-\mathbf{q}_{1}-\mathbf{l}^{\prime}}^{v, 3,}\right] \Delta\left\langle a_{\mathbf{k}-\mathbf{q}_{1}, c}^{\dagger} a_{\mathbf{l}, v^{\prime \prime}}^{\dagger} a_{\mathbf{l}+\mathbf{l}^{\prime}, c^{\prime \prime}} a_{\mathbf{k}-\mathbf{q}_{1}-\mathbf{l}^{\prime}, 2}\right\rangle \Delta\left\langle a_{\mathbf{p}, 1}^{\dagger} a_{\mathbf{k}^{\prime}, v^{\prime}}^{\dagger} a_{\mathbf{k}^{\prime}-\mathbf{q}_{2}, c^{\prime}} a_{\mathbf{p}+\mathbf{q}_{2}, 3}\right\rangle \\
& -\left[V_{\mathbf{k}, \mathbf{p}, \mathbf{k}+\mathbf{l}^{\prime}, \mathbf{p}-\mathbf{l}^{\prime}}^{v, 1,3}-V_{\mathbf{k}, \mathbf{p}, \mathbf{p}-\mathbf{l}^{\prime}, \mathbf{k}+\mathbf{l}^{\prime}}^{v, 1,3,}\right] \Delta\left\langle a_{\mathbf{k}-\mathbf{q}_{1}, c}^{\dagger} a_{\mathbf{k}^{\prime}, v^{\prime}}^{\dagger} a_{\mathbf{k}^{\prime}-\mathbf{q}_{2}, c^{\prime}} a_{\mathbf{k}+\mathbf{l}^{\prime}, 2}\right\rangle \Delta\left\langle a_{\mathbf{p}, 1}^{\dagger} a_{\mathbf{1}, v^{\prime \prime}}^{\dagger} a_{\mathbf{1}+\mathbf{l}^{\prime}, c^{\prime \prime}} a_{\mathbf{p}-\mathbf{l}^{\prime}, 3}\right\rangle \\
& +\left[V_{\mathbf{p}, \mathbf{l}+\mathbf{l}^{\prime}+\mathbf{q}_{1}, \mathbf{p}+\mathbf{q}_{2}, \mathbf{l}}^{1,2, v^{\prime \prime}}-V_{\mathbf{l}+\mathbf{l}^{\prime}+\mathbf{q}_{1}, \mathbf{p}, \mathbf{p}+\mathbf{q}_{2}, \mathbf{l}}^{2,1,3, v^{\prime \prime}}\right] \Delta\left\langle a_{\mathbf{l}+\mathbf{l}^{\prime}+\mathbf{q}_{1}, 2}^{\dagger} a_{\mathbf{k}-\mathbf{q}_{1}, c}^{\dagger} a_{\mathbf{k}, v} a_{\mathbf{l}+\mathbf{l}^{\prime}, c^{\prime \prime}}\right\rangle \Delta\left\langle a_{\mathbf{p}, 1}^{\dagger} a_{\mathbf{k}^{\prime}, v^{\prime}}^{\dagger} a_{\mathbf{k}^{\prime}-\mathbf{q}_{2}, c^{\prime}} a_{\mathbf{p}+\mathbf{q}_{2}, 3}\right\rangle \\
& \left.-\left[V_{\mathbf{l}+\mathbf{1}^{\prime}, \mathbf{p}, \mathbf{l}-\mathbf{q}_{1}, \mathbf{p}+\mathbf{q}_{2}}^{c^{\prime \prime}, 1,3}-V_{\mathbf{l}+\mathbf{l}^{\prime}, \mathbf{p}, \mathbf{p}+\mathbf{q}_{2}, \mathbf{l}-\mathbf{q}_{1}}^{c^{\prime \prime}, 1,2}\right] \Delta\left\langle a_{\mathbf{k}-\mathbf{q}_{1}, c}^{\dagger} a_{\mathbf{l}, v^{\prime \prime}}^{\dagger} a_{\mathbf{l}-\mathbf{q}_{1}, 2} a_{\mathbf{k}, v}\right\rangle \Delta\left\langle a_{\mathbf{k}^{\prime}, v^{\prime}}^{\dagger} a_{\mathbf{p}, 1}^{\dagger} a_{\mathbf{p}+\mathbf{q}_{2}, 3} a_{\mathbf{k}^{\prime}-\mathbf{q}_{2}, c^{\prime}}\right\rangle\right\} \\
& +\sum_{1}\left[V_{\mathbf{l}+\mathbf{1}^{\prime}, \mathbf{k}^{\prime}-\mathbf{1}^{\prime}, \mathbf{l}, \mathbf{k}^{\prime}}^{c^{\prime \prime}}-V_{\mathbf{k}^{\prime}-\mathbf{1}^{\prime}, \mathbf{l}+\mathbf{1}^{\prime}, \mathbf{1}, \mathbf{k}^{\prime}}^{1, c^{\prime \prime}, v^{\prime \prime}}\right] \Delta\left\langle a_{\mathbf{k}^{\prime}-\mathbf{l}^{\prime}, 1}^{\dagger} a_{\mathbf{k}-\mathbf{q}_{1}, c}^{\dagger} a_{\mathbf{k}, v} a_{\mathbf{k}^{\prime}-\mathbf{q}_{2}, c^{\prime}}\right\rangle \\
& +\sum_{1}\left[V_{\mathbf{l}+\mathbf{l}^{\prime}, \mathbf{k}^{\prime}-\mathbf{q}_{2}, \mathbf{l}+\mathbf{l}^{\prime}-\mathbf{q}_{2}, \mathbf{k}^{\prime}}^{c^{\prime \prime}, c^{\prime}, 1, v^{\prime}}-V_{\mathbf{k}^{\prime}-\mathbf{q}_{2}, \mathbf{l}+\mathbf{l}^{\prime}, \mathbf{l}+\mathbf{l}^{\prime}-\mathbf{q}_{2}, \mathbf{k}^{\prime}}^{c^{\prime}, c^{\prime \prime}, 1, v^{\prime}}\right] \Delta\left\langle a_{\mathbf{k}-\mathbf{q}_{1}, c}^{\dagger} a_{\mathbf{l}, v^{\prime \prime}}^{\dagger} a_{\mathbf{k}, v} a_{\mathbf{1}+\mathbf{l}^{\prime}-\mathbf{q}_{2}, 1}\right\rangle \\
& +\sum_{1}\left[V_{\mathbf{k}^{\prime}-\mathbf{q}_{2}, \mathbf{l}+\mathbf{q}_{2}, \mathbf{l}, \mathbf{k}^{\prime}}^{c^{\prime}, 1, v^{\prime \prime}, v^{\prime}}-V_{\mathbf{l}+\mathbf{q}_{2}, \mathbf{k}^{\prime}-\mathbf{q}_{2}, \mathbf{l}, \mathbf{k}^{\prime}}^{1, c^{\prime}, v^{\prime \prime}, v^{\prime}}\right] \Delta\left\langle a_{\mathbf{l}+\mathbf{q}_{2}, 1}^{\dagger} a_{\mathbf{k}-\mathbf{q}_{1}, c}^{\dagger} a_{\mathbf{1}+\mathbf{l}^{\prime}, c^{\prime \prime}} a_{\mathbf{k}, v}\right\rangle \\
& +\sum_{1}\left[V_{\mathbf{l}+\mathbf{l}^{\prime}, \mathbf{k}^{\prime}-\mathbf{q}_{2}, \mathbf{k}^{\prime}+\mathbf{l}^{\prime}-\mathbf{q}_{2}, \mathbf{l}}^{c^{\prime \prime}, c^{\prime}, 1, v^{\prime \prime}}-V_{\mathbf{k}^{\prime}-\mathbf{q}_{2}, \mathbf{l}+\mathbf{l}^{\prime}, \mathbf{k}^{\prime}+\mathbf{l}^{\prime}-\mathbf{q}_{2}, \mathbf{l}}^{c^{\prime}, c^{\prime \prime}, 1, v^{\prime \prime}}\right] \Delta\left\langle a_{\mathbf{k}^{\prime}, v^{\prime}}^{\dagger} a_{\mathbf{k}-\mathbf{q}_{1}, c^{\dagger}}^{\dagger} a_{\mathbf{k}, v} a_{\mathbf{k}^{\prime}+\mathbf{l}^{\prime}-\mathbf{q}_{2}, 1}\right\rangle .
\end{aligned}
$$

The above result is inserted into Eq. (S22). After reintroducing two-particle operators, using the orthonor- mality of wave functions as well as the Bethe-Salpeter equation S14 and discarding all doublets that are not density-like, we arrive at: 


$$
\begin{aligned}
& i \hbar \frac{d}{d t} \Delta\left\langle X_{\alpha, \mathbf{q}_{1}}^{\dagger} X_{\beta, \mathbf{q}_{2}} X_{\delta,-\mathbf{1}^{\prime}}\right\rangle \\
& =\left(E_{\beta, \mathbf{q}_{2}}+E_{\delta,-\mathbf{1}^{\prime}}-E_{\alpha, \mathbf{q}_{1}}\right) \Delta\left\langle X_{\alpha, \mathbf{q}_{1}}^{\dagger} X_{\beta, \mathbf{q}_{2}} X_{\delta,-\mathbf{1}^{\prime}}\right\rangle \\
& +\frac{1}{\mathcal{A}}\left\{\left[\hat{V}_{\alpha, \beta, \delta, \mathbf{q}_{1}, \mathbf{l}^{\prime}}^{(1), X}-\hat{V}_{\alpha, \beta, \delta, \mathbf{q}_{1}, \mathbf{l}^{\prime}}^{(1), D}\right] n_{\beta, \mathbf{q}_{2}} n_{\delta,-\mathbf{l}^{\prime}}+\left[\hat{V}_{\alpha, \beta, \delta, \mathbf{q}_{2}, \mathbf{l}^{\prime}}^{(2), X}-\hat{V}_{\alpha, \beta, \delta, \mathbf{q}_{2}, \mathbf{l}^{\prime}}^{(2), D}\right] n_{\alpha, \mathbf{q}_{1}} n_{\delta,-\mathbf{l}^{\prime}}\right. \\
& +\left[\hat{V}_{\alpha, \delta, \beta, \mathbf{q}_{1},-\mathbf{q}_{2}}^{(1), X}-\hat{V}_{\alpha, \delta, \beta, \mathbf{q}_{1},-\mathbf{q}_{2}}^{(1), D}\right] n_{\beta, \mathbf{q}_{2}} n_{\delta,-\mathbf{l}^{\prime}}-\left[\hat{V}_{\alpha, \beta, \delta, \mathbf{q}_{1}, \mathbf{1}^{\prime}}^{(1), X}-\hat{V}_{\alpha, \beta, \delta, \mathbf{q}_{1}, \mathbf{1}^{\prime}}^{(1), D}\right] n_{\alpha, \mathbf{q}_{1}} n_{\delta,-\mathbf{l}^{\prime}} \\
& -\left[\hat{V}_{\alpha, \beta, \delta, \mathbf{q}_{2}, \mathbf{1}^{\prime}}^{(2), X}-\hat{V}_{\alpha, \beta, \delta, \mathbf{q}_{2}, \mathbf{l}^{\prime}}^{(2), D}\right] n_{\beta, \mathbf{q}_{2}} n_{\delta,-\mathbf{l}^{\prime}}-\left[\hat{V}_{\alpha, \delta, \beta,-\mathbf{l}^{\prime},-\mathbf{q}_{2}}^{(2), X}-\hat{V}_{\alpha, \delta, \beta,-\mathbf{l}^{\prime},-\mathbf{q}_{2}}^{(2), D}\right] n_{\beta, \mathbf{q}_{2}} n_{\delta,-\mathbf{l}^{\prime}} \\
& +\left[\hat{V}_{\alpha, \delta, \beta,-\mathbf{l}^{\prime},-\mathbf{q}_{2}}^{(2), X}-\hat{V}_{\alpha, \delta, \beta,-\mathbf{1}^{\prime},-\mathbf{q}_{2}}^{(2), D}\right] n_{\alpha, \mathbf{q}_{1}} n_{\beta, \mathbf{q}_{2}}-\left[\hat{V}_{\alpha, \delta, \beta, \mathbf{q}_{1},-\mathbf{q}_{2}}^{(1), X}-\hat{V}_{\alpha, \delta, \beta, \mathbf{q}_{1},-\mathbf{q}_{2}}^{(1), D}\right] n_{\alpha, \mathbf{q}_{1}} n_{\beta, \mathbf{q}_{2}} \\
& +\left[\hat{V}_{\alpha, \beta, \delta, \mathbf{q}_{2}, \mathbf{l}^{\prime}}^{(2), X}-\hat{V}_{\alpha, \beta, \delta, \mathbf{q}_{2}, \mathbf{1}^{\prime}}^{(2), D}\right] n_{\alpha, \mathbf{q}_{1}}-\left[\hat{V}_{\alpha, \delta, \beta, \mathbf{q}_{1},-\mathbf{q}_{2}}^{(1), X}-\hat{V}_{\alpha, \delta, \beta, \mathbf{q}_{1},-\mathbf{q}_{2}}^{(1), D}\right] n_{\alpha, \mathbf{q}_{1}} \\
& \left.+\left[\hat{V}_{\alpha, \delta, \beta,-\mathbf{l}^{\prime},-\mathbf{q}_{2}}^{(2), X}-\hat{V}_{\alpha, \delta, \beta,-\mathbf{1}^{\prime},-\mathbf{q}_{2}}^{(2), D}\right] n_{\alpha, \mathbf{q}_{1}}-\left[\hat{V}_{\alpha, \beta, \delta, \mathbf{q}_{1}, \mathbf{1}^{\prime}}^{(1), X}-\hat{V}_{\alpha, \beta, \delta, \mathbf{q}_{1}, \mathbf{1}^{\prime}}^{(1), D}\right] n_{\alpha, \mathbf{q}_{1}}\right\} .
\end{aligned}
$$

Note that by focussing on terms that contain exciton populations, the Coulomb matrix elements are automatically forced to Auger-like band index combinations containing one inter-gap scattering process. This means that on the triplet level, the only Coulomb interaction processes between exciton densities are of EEA type. Any scattering processes that conserve the number of excitons would involve terms that are of third order in the exciton density, which require the factorization of six-particle expectation values and therefore appear on a higher level of cluster expansion.

We eliminate the triplets by adding a phenomenological damping $\Gamma$ to the oscillating terms and applying the Markov approximation [25], which yields algebraic expressions:

$\Delta\left\langle X_{\alpha, \mathbf{q}_{1}}^{\dagger} X_{\beta, \mathbf{q}_{2}} X_{\delta,-\mathbf{1}^{\prime}}\right\rangle(t) \approx \frac{-K_{\alpha, \beta, \delta, \mathbf{q}_{1}, \mathbf{q}_{2},-\mathbf{1}^{\prime}}(t)}{E_{\beta, \mathbf{q}_{2}}+E_{\delta,-\mathbf{1}^{\prime}}-E_{\alpha, \mathbf{q}_{1}}-i \Gamma}$,

where $K(t)$ denotes the inhomogeneity on the RHS of Eq. (S26). The triplets in Markov approximation are inserted into Eq. (S21). After combining the terms and using the relation $\operatorname{Im} z^{*}=-\operatorname{Im} z$, we arrive at the final result:

$$
\begin{aligned}
& \frac{d}{d t} n_{\alpha, \mathbf{q}}=\frac{1}{\mathcal{A}^{2}} \sum_{\mathbf{l}} \frac{2}{\hbar} \operatorname{Im}\{ \\
& \sum_{\beta \delta} V_{\mathbf{q},-\mathbf{l}}^{D, \alpha, \beta, \delta} \frac{1}{E_{\alpha, \mathbf{q}}-E_{\beta, \mathbf{q}-\mathbf{1}}-E_{\delta, \mathbf{1}}-i \Gamma} \times \\
& \left\{\left(V_{\mathbf{q},-\mathbf{l}}^{D, \alpha, \beta}-V_{\mathbf{q},-1}^{X, \alpha, \beta, \delta}\right)^{*}\left(n_{\beta, \mathbf{q}-\mathbf{l}} n_{\delta, \mathbf{l}}-n_{\alpha, \mathbf{q}}\left(n_{\delta, \mathbf{l}}+1\right)\right)\right. \\
& \left.+\left(V_{\mathbf{q},-\mathbf{q}+\mathbf{l}}^{D, \alpha, \beta}-V_{\mathbf{q},-\mathbf{q}+\mathbf{l}}^{X, \alpha, \beta}\right)^{*}\left(n_{\beta, \mathbf{q}-\mathbf{l}} n_{\delta, \mathbf{l}}-n_{\alpha, \mathbf{q}}\left(n_{\beta, \mathbf{q}-\mathbf{l}}+1\right)\right)\right\} \\
& -\sum_{\beta \delta} V_{\mathbf{q}+\mathbf{1},-\mathbf{1}}^{D, \beta, \delta} \frac{1}{E_{\beta, \mathbf{q}+\mathbf{l}}-E_{\alpha, \mathbf{q}}-E_{\delta, \mathbf{1}}-i \Gamma} \times \\
& \left\{\left(V_{\mathbf{q}+\mathbf{1},-\mathbf{l}}^{D, \beta, \delta}-V_{\mathbf{q}+\mathbf{1},-1}^{X, \beta, \delta}\right)^{*}\left(n_{\alpha, \mathbf{q}} n_{\delta, \mathbf{1}}-n_{\beta, \mathbf{q}+\mathbf{1}}\left(n_{\delta, \mathbf{l}}+1\right)\right)\right. \\
& \left.+\left(V_{\mathbf{q}+\mathbf{1},-\mathbf{q}}^{D, \delta}-V_{\mathbf{q}+\mathbf{l},-\mathbf{q}}^{X, \beta, \alpha}\right)^{*}\left(n_{\alpha, \mathbf{q}} n_{\delta, \mathbf{l}}-n_{\beta, \mathbf{q}+\mathbf{l}}\left(n_{\alpha, \mathbf{q}}+1\right)\right)\right\} \\
& -\sum_{\beta \delta} V_{\mathbf{q}+\mathbf{1},-1}^{X, \beta, \delta} \frac{1}{E_{\beta, \mathbf{q}+\mathbf{l}}-E_{\alpha, \mathbf{q}}-E_{\delta, \mathbf{1}}-i \Gamma} \times \\
& \left\{\left(V_{\mathbf{q}+\mathbf{1},-\mathbf{l}}^{X, \beta, \delta}-V_{\mathbf{q}+\mathbf{1},-\mathbf{l}}^{D, \beta, \delta}\right)^{*}\left(n_{\alpha, \mathbf{q}} n_{\delta, \mathbf{1}}-n_{\beta, \mathbf{q}+\mathbf{l}}\left(n_{\delta, \mathbf{1}}+1\right)\right)\right. \\
& \left.\left.+\left(V_{\mathbf{q}+\mathbf{1},-\mathbf{q}}^{X, \beta, \alpha}-V_{\mathbf{q}+\mathbf{1},-\mathbf{q}}^{D, \beta, \delta}\right)^{*}\left(n_{\alpha, \mathbf{q}} n_{\delta, \mathbf{l}}-n_{\beta, \mathbf{q}+\mathbf{l}}\left(n_{\alpha, \mathbf{q}}+1\right)\right)\right\}\right\} .
\end{aligned}
$$

There are three scattering channels for the exciton in state $|\alpha, \boldsymbol{q}\rangle$ corresponding to the different roles that the exciton can play in an EEA process, see Fig. S4. Each channel contains a sum of two terms corresponding to the exhange of a whole exciton, e.g. states $|\beta, \mathbf{q}-\mathbf{l}\rangle$ and $|\delta, \mathbf{l}\rangle$ for the first channel, due to the bosonic symmetry of triplets. The bosonic symmetry is combined with fermionic symmetries corresponding to the exchange of an electron or hole between two excitons, which becomes visible in the Bloch state representation of triplets, e.g. in Eq. (S25). Note that the interpretation of terms is complicated by these quantum symmetries, since particles can change roles in the scattering process. In particular, the EOM do not have the form of a Boltzmann equa- 

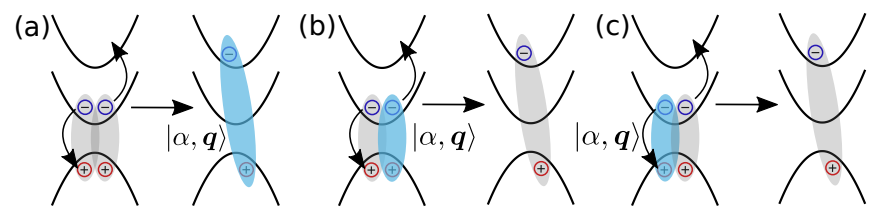

FIG. S4. Different roles of exciton state $|\alpha, \boldsymbol{q}\rangle$ in EEA process. (a): As high-energy target state. (b): As 1s-exciton state that takes up the excess energy and momentum from the annihilated exciton. (c) As the annihilated 1s-exciton.

tion with terms like $V_{\mathbf{q},-1}^{D, \beta, \delta}\left(V_{\mathbf{q},-1}^{D, \beta, \delta}-V_{\mathbf{q},-\mathbf{l}}^{X, \alpha, \beta, \delta}\right)^{*}((1+$ $\left.\left.n_{\alpha, \mathbf{q}}\right) n_{\beta, \mathbf{q}-\mathbf{1}} n_{\delta, \mathbf{1}}-n_{\alpha, \mathbf{q}}\left(1+n_{\beta, \mathbf{q}-\mathbf{l}}\right)\left(1+n_{\delta, \mathbf{l}}\right)\right)$. Even though we have formulated our theory in terms of exciton or two-particle quantities, it has been derived from a fundamental electron-electron Coulomb interaction. Hence it knows about the compound nature of excitons as bound electron-hole pairs. In this context, we compare the fully microscopic theory to a purely bosonic effective theory in the next section.

\section{Bosonic EEA theory}

In the following, we set up an excitonic Hamiltonian to investigate if EEA can be understood in an effective bosonic picture. The Hamiltonian that describes the process sketched in Fig. $\mathrm{S} 4$ is:

$$
\begin{array}{r}
H=\sum_{\alpha, \beta, \delta, \mathbf{q}, \mathbf{l}}\left(V_{\mathbf{q}, \mathbf{l}}^{\alpha, \beta, \delta} X_{\delta,-\mathbf{l}}^{\dagger} X_{\beta, \mathbf{q}+\mathbf{l}}^{\dagger} X_{\alpha, \mathbf{q}}\right. \\
\left.+\left(V_{\mathbf{q}, \mathbf{l}}^{\alpha, \beta, \delta}\right)^{*} X_{\alpha, \mathbf{q}}^{\dagger} X_{\beta, \mathbf{q}+\mathbf{l}} X_{\delta,-\mathbf{l}}\right)
\end{array}
$$

with effective exciton-exciton interaction matrix elements $V_{\mathbf{q}, 1}^{\alpha, \beta, \delta}$ and bosonic operators that fulfill $\left[X_{\alpha, \mathbf{q}}, X_{\beta, \mathbf{q}^{\prime}}^{\dagger}\right]=$ $\delta_{\alpha \beta} \delta_{\mathbf{q}, \mathbf{q}^{\prime}}$. As in the previous section, we use the cluster expansion technique to obtain a closed set of dynamical equations. First, we derive EOM for triplets (without the trivial oscillating part), where we discard all factorizations that contain non-density-like correlation functions:

$$
\begin{aligned}
& \left.i \hbar \frac{d}{d t} \Delta\left\langle X_{\alpha, \mathbf{q}_{1}}^{\dagger} X_{\beta, \mathbf{q}_{2}} X_{\delta,-1}\right\rangle\right|_{\mathrm{EEA}} \\
= & \left(V_{\mathbf{q}_{1}, \mathbf{1}}^{\alpha, \beta, \delta}+V_{\mathbf{q}_{1},-\mathbf{q}_{2}}^{\alpha, \delta, \beta}\right) \times \\
& \left\{n_{\alpha, \mathbf{q}_{1}} n_{\delta,-1}-n_{\beta, \mathbf{q}_{2}} n_{\delta,-1}+n_{\alpha, \mathbf{q}_{1}}\left(1+n_{\beta, \mathbf{q}_{2}}\right)\right\} \\
= & \left(V_{\mathbf{q}_{1}, \mathbf{l}}^{\alpha, \beta, \delta}+V_{\mathbf{q}_{1},-\mathbf{q}_{2}}^{\alpha, \delta, \beta}\right) \times \\
& \left\{n_{\alpha, \mathbf{q}_{1}}\left(1+n_{\delta,-1}\right)\left(1+n_{\beta, \mathbf{q}_{2}}\right)-\left(1+n_{\alpha, \mathbf{q}_{1}}\right) n_{\delta,-1} n_{\beta, \mathbf{q}_{2}}\right\} .
\end{aligned}
$$

For the exciton densities we obtain:

$$
\begin{gathered}
\frac{d}{d t} n_{\alpha, \mathbf{q}}=\sum_{\beta, \delta, \mathbf{l}} \times \\
\frac{2}{\hbar} \operatorname{Im}\left\{\left(V_{\mathbf{q}, \mathbf{l}}^{\alpha, \beta}\right)^{*} \Delta\left\langle X_{\alpha, \mathbf{q}}^{\dagger} X_{\beta, \mathbf{q}+\mathbf{l}} X_{\delta,-\mathbf{l}}\right\rangle\right. \\
\left.+\left(V_{\mathbf{q}+\mathbf{l},-\mathbf{l}}^{\beta, \alpha}+V_{\mathbf{q}+\mathbf{l},-\mathbf{q}}^{\beta, \delta, \alpha}\right)\left(\Delta\left\langle X_{\beta, \mathbf{q}+\mathbf{l}}^{\dagger} X_{\delta, \mathbf{l}} X_{\alpha, \mathbf{q}}\right\rangle\right)^{*}\right\} .
\end{gathered}
$$

Inserting the triplets in Markov approximation yields:

$$
\begin{aligned}
& \frac{d}{d t} n_{\alpha, \mathbf{q}}=\sum_{\beta, \delta, \mathbf{1}} \frac{2}{\hbar} \operatorname{Im}\{ \\
& V_{\mathbf{q},-\mathbf{l}}^{\alpha, \beta, \delta}\left(V_{\mathbf{q},-\mathbf{l}}^{\alpha, \beta, \delta}+V_{\mathbf{q},-\mathbf{q}+\mathbf{l}}^{\alpha, \delta, \beta}\right)^{*} \frac{1}{E_{\alpha, \mathbf{q}}-E_{\beta, \mathbf{q}-\mathbf{l}}-E_{\delta, \mathbf{1}}-i \Gamma} \times \\
& \left\{\left(1+n_{\alpha, \mathbf{q}}\right) n_{\delta, \mathbf{l}} n_{\beta, \mathbf{q}-\mathbf{l}}-n_{\alpha, \mathbf{q}}\left(1+n_{\delta, \mathbf{l}}\right)\left(1+n_{\beta, \mathbf{q}-\mathbf{l}}\right)\right\} \\
& -V_{\mathbf{q}+\mathbf{l},-\mathbf{l}}^{\beta, \alpha}\left(V_{\mathbf{q}+\mathbf{l},-\mathbf{l}}^{\beta, \alpha, \delta}+V_{\mathbf{q}+\mathbf{l},-\mathbf{q}}^{\beta, \delta, \alpha}\right)^{*} \frac{1}{E_{\beta, \mathbf{q}+\mathbf{l}}-E_{\alpha, \mathbf{q}}-E_{\delta, \mathbf{l}}-i \Gamma} \times \\
& \left\{\left(1+n_{\beta, \mathbf{q}+\mathbf{l}}\right) n_{\delta, \mathbf{l}} n_{\alpha, \mathbf{q}}-n_{\beta, \mathbf{q}+\mathbf{l}}\left(1+n_{\delta, \mathbf{l}}\right)\left(1+n_{\alpha, \mathbf{q}}\right)\right\} \\
& -V_{\mathbf{q}+\mathbf{l},-\mathbf{q}}^{\beta, \delta}\left(V_{\mathbf{q}+\mathbf{l},-\mathbf{q}}^{\beta, \delta, \alpha}+V_{\mathbf{q}+\mathbf{l},-\mathbf{l}}^{\beta, \alpha}\right)^{*} \frac{1}{E_{\beta, \mathbf{q}+\mathbf{l}}-E_{\alpha, \mathbf{q}}-E_{\delta, \mathbf{l}}-i \Gamma} \times \\
& \left.\left\{\left(1+n_{\beta, \mathbf{q}+\mathbf{l}}\right) n_{\delta, \mathbf{l}} n_{\alpha, \mathbf{q}}-n_{\beta, \mathbf{q}+\mathbf{l}}\left(1+n_{\delta, \mathbf{l}}\right)\left(1+n_{\alpha, \mathbf{q}}\right)\right\}\right\} \text {. }
\end{aligned}
$$

The scattering integrals on the RHS have to be compared to those in Eq. S28 derived from the fundamental electron-electron interaction Hamiltonian. Both equations exhibit three main scattering channels, with two terms for each channel reflecting the indistinguishablity of the scattering excitons. In Eq. (S32), the two terms are given by sums over matrix elements such as $V_{\mathbf{q},-1}^{\alpha, \beta, \delta}+V_{\mathbf{q},-\mathbf{q}+\mathbf{l}}^{\alpha, \delta}$. Unlike the fully microscopic EOM, the bosonic EOM have the form of a Boltzmann equation in the sense that population factors $n$ and $1+n$ can be clearly assigned to scattering out of an exciton state and scattering into an exciton state, respectively. The assignment of in- and out-scattering is consistent with the (approximate) energy conservation as well as the exciton-exciton interaction matrix elements, where $V_{\mathbf{q},-\mathbf{l}}^{\alpha, \beta, \delta}$ belongs to scattering between $|\alpha, \mathbf{q}\rangle$ and $|\beta, \mathbf{q}+\mathbf{l}\rangle$, $|\delta,-\mathbf{l}\rangle$. The key difference is that the purely bosonic theory can not account for the exchange of a single electron or hole between two excitons (reflected by a change from $V_{\mathbf{q},-1}^{D, \alpha, \beta, \delta}$ to $\left.-V_{\mathbf{q},-\mathbf{l}}^{X, \alpha, \beta, \delta}\right)$, but only of an exciton as a whole $\left(V_{\mathbf{q},-1}^{\alpha, \beta, \delta}\right.$ to $\left.V_{\mathbf{q},-\mathbf{q}+\mathbf{l}}^{\alpha, \delta}\right)$. To clarify this, we compare the EOM for triplets as derived from the two Hamiltonians term by 
term:

$$
\begin{aligned}
& \left.i \hbar \frac{d}{d t} \Delta\left\langle X_{\alpha, \mathbf{q}_{1}}^{\dagger} X_{\beta, \mathbf{q}_{2}} X_{\delta,-1}\right\rangle\right|_{\text {bosonic }} \\
& =V_{\mathbf{q}_{1}, \mathbf{1}}^{\alpha, \beta, \delta}\{\underbrace{n_{\alpha, \mathbf{q}_{1}} n_{\delta,-1}}_{\# 1}-\underbrace{n_{\beta, \mathbf{q}_{2}} n_{\delta,-1}}_{\# 2}+\underbrace{n_{\alpha, \mathbf{q}_{1}}}_{\# 3}+\underbrace{n_{\alpha, \mathbf{q}_{1}} n_{\beta, \mathbf{q}_{2}}}_{\# 4}\} \\
& +V_{\mathbf{q}_{1},-\mathbf{q}_{2}}^{\alpha, \delta, \beta}\{\underbrace{n_{\alpha, \mathbf{q}_{1}} n_{\delta,-1}}_{\# 5}-\underbrace{n_{\beta, \mathbf{q}_{2}} n_{\delta,-1}}_{\# 6}+\underbrace{n_{\alpha, \mathbf{q}_{1}}}_{\# 7}+\underbrace{n_{\alpha, \mathbf{q}_{1}} n_{\beta, \mathbf{q}_{2}}}_{\# 8}\}, \\
& \left.i \hbar \frac{d}{d t} \Delta\left\langle X_{\alpha, \mathbf{q}_{1}}^{\dagger} X_{\beta, \mathbf{q}_{2}} X_{\delta,-1}\right\rangle\right|_{\text {full }} \\
& =V_{\mathbf{q}_{1}, \mathbf{l}}^{D, \alpha, \beta, \delta} \underbrace{n_{\alpha, \mathbf{q}_{1}} n_{\delta,-1}}_{\# 1}-\left(V_{\mathbf{q}_{1}, \mathbf{l}}^{D, \alpha, \beta, \delta}-V_{\mathbf{q}_{1}, \mathbf{l}}^{X, \alpha, \beta, \delta}\right) \underbrace{n_{\beta, \mathbf{q}_{2}} n_{\delta,-1}}_{\# 2} \\
& +\left(V_{\mathbf{q}_{1}, \mathbf{l}}^{D, \alpha, \beta, \delta}-V_{\mathbf{q}_{1}, \mathbf{l}}^{X, \alpha, \beta}\right) \underbrace{n_{\alpha, \mathbf{q}_{1}}}_{\# 3}-V_{\mathbf{q}_{1},-\mathbf{q}_{2}}^{X, \alpha, \delta, \beta} \underbrace{n_{\alpha, \mathbf{q}_{1}} n_{\beta, \mathbf{q}_{2}}}_{\# 4} \\
& -V_{\mathbf{q}_{1}, \mathbf{l}}^{X, \alpha, \beta} \underbrace{n_{\alpha, \mathbf{q}_{1}} n_{\delta,-1}}_{\# 5}-\left(V_{\mathbf{q}_{1},-\mathbf{q}_{2}}^{D, \alpha, \delta, \beta}-V_{\mathbf{q}_{1},-\mathbf{q}_{2}}^{X, \alpha, \delta, \beta}\right) \underbrace{n_{\beta, \mathbf{q}_{2}} n_{\delta,-1}}_{\# 6} \\
& +\left(V_{\mathbf{q}_{1},-\mathbf{q}_{2}}^{D, \alpha, \delta, \beta}-V_{\mathbf{q}_{1},-\mathbf{q}_{2}}^{X, \alpha, \delta}\right) \underbrace{n_{\alpha, \mathbf{q}_{1}}}_{\# 7}+V_{\mathbf{q}_{1},-\mathbf{q}_{2}}^{D, \alpha, \delta} \underbrace{n_{\alpha, \mathbf{q}_{1}} n_{\beta, \mathbf{q}_{2}}}_{\# 8} .
\end{aligned}
$$

While in the purely bosonic picture, terms can be collected such that Boltzmann-like population factors emerge, this is not possible in the full theory due to the exchange matrix elements. The possible exchange of fermionic constituents of excitons is what inhibits the fully microscopic EOM to be cast into the form of a Boltzmann equation with effective exciton-exciton interaction matrix elements. This is consistent with the more general discussion by $M$. Combescot et al. that it is not possible to formulate a closed expression for an effective exciton-exciton interaction potential [29].

We finally compare the two pictures in the limiting case where the exciton distribution is close to equilibrium due to fast relaxation processes. Focussing on the EOM of 1s-exciton populations and neglecting the populations of high-energy states, we find:

$$
\begin{aligned}
& \left.\frac{d}{d t} n_{\alpha, \mathbf{q}}\right|_{\text {full }}=-\frac{1}{\mathcal{A}^{2}} \sum_{\mathbf{l}} \sum_{\beta \delta} \frac{2}{\hbar} \times \\
& \operatorname{Im}\left\{\left(V_{\mathbf{q}+\mathbf{l},-\mathbf{l}}^{D, \beta, \alpha, \delta}-V_{\mathbf{q}+\mathbf{l},-\mathbf{l}}^{X, \beta, \alpha, \delta}\right) \times\right. \\
& \left(\left(V_{\mathbf{q}+\mathbf{l},-\mathbf{l}}^{D, \beta, \alpha, \delta}-V_{\mathbf{q}+\mathbf{l},-\mathbf{l}}^{X, \beta, \alpha}\right)^{*}+\left(V_{\mathbf{q}+\mathbf{l},-\mathbf{q}}^{D, \beta, \delta, \alpha}-V_{\mathbf{q}+\mathbf{l},-\mathbf{q}}^{X, \beta, \delta, \alpha}\right) *\right. \\
& \left.\frac{1}{E_{\beta, \mathbf{q}+\mathbf{l}}-E_{\alpha, \mathbf{q}}-E_{\delta, \mathbf{l}}-i \Gamma}\right\} n_{\alpha, \mathbf{q}} n_{\delta, \mathbf{l}}
\end{aligned}
$$

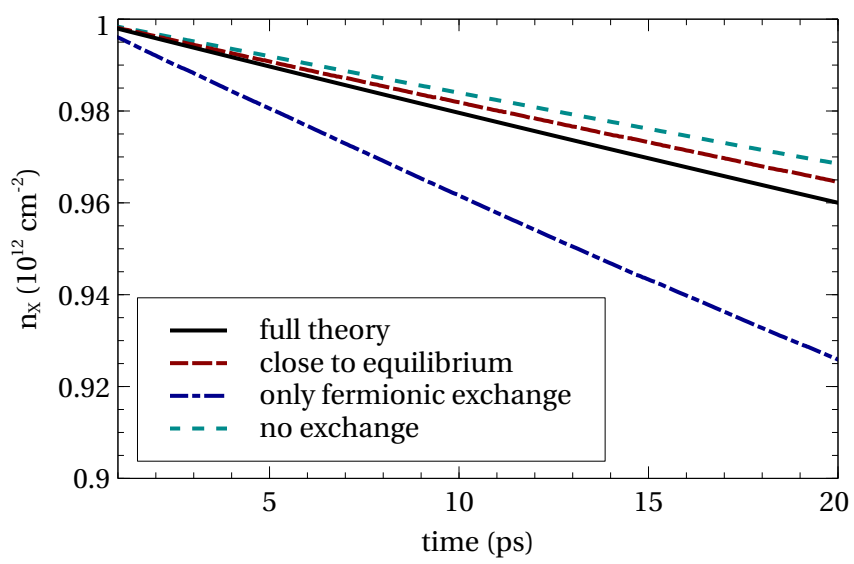

FIG. S5. Decay of the total exciton density in hBNencapsulated $\mathrm{MoS}_{2}$ at $T=300 \mathrm{~K}$ obtained from different approximations to our EEA theory. We compare the full theory (Eq. (S28) ) to the simplified EOM in the close-to-equilibrium limit (Eq. (S34). Further approximations that lead to an equivalence of Eqs. S34 and (S35) are performed by including only fermionic exchange and by neglecting all exchange effects. The corresponding EEA coefficients are given in the text.

and

$$
\begin{gathered}
\left.\frac{d}{d t} n_{\alpha, \mathbf{q}}\right|_{\text {bosonic }}=-\frac{1}{\mathcal{A}^{2}} \sum_{\mathbf{l}} \sum_{\beta \delta} \frac{2}{\hbar}\left|V_{\mathbf{q}+\mathbf{l},-\mathbf{l}}^{\beta, \alpha, \delta}+V_{\mathbf{q}+\mathbf{l},-\mathbf{q}}^{\beta, \delta, \alpha}\right|^{2} \times \\
\operatorname{Im}\left\{\frac{1}{E_{\beta, \mathbf{q}+\mathbf{l}}-E_{\alpha, \mathbf{q}}-E_{\delta, \mathbf{1}}-i \Gamma}\right\} n_{\alpha, \mathbf{q}} n_{\delta, \mathbf{l}} .
\end{gathered}
$$

While the effective bosonic picture allows to identify the modulus square of an exciton-exciton interaction matrix element that obeys bosonic symmetry, this is still not possible in the fully microscopic theory.

An equivalence between the full theory and the bosonic theory can only be obtained when the exchange of whole excitons is neglected, which amounts to discarding the Coulomb matrix elements $V_{\mathbf{q}+\mathbf{1},-\mathbf{q}}^{\beta, \delta}$ in Eqs. S34 and (S35). Then the effective bosonic interaction matrix element can be identified as $V_{\mathbf{q}+\mathbf{1},-1}^{D, \beta, \alpha}-V_{\mathbf{q}+\mathbf{l},-1}^{X, \beta, \alpha}$, which means that fermionic exchange can still be included in this case. In Fig. S5, we demonstrate the quantitative effect of this approximation. First of all, when reducing the full theory to the close-to-equilibrium case, the EEA coefficient decreases from $0.21 \times 10^{-3} \mathrm{~cm}^{2} \mathrm{~s}^{-1}$ to $0.18 \times 10^{-3}$ $\mathrm{cm}^{2} \mathrm{~s}^{-1}$. Neglecting the exchange of full excitons leads to an increase of the coefficient to $0.40 \times 10^{-3} \mathrm{~cm}^{2} \mathrm{~s}^{-1}$. The coefficient is reduced again to $0.16 \times 10^{-3} \mathrm{~cm}^{2} \mathrm{~s}^{-1}$ in the absence of all exchange processes, which means that EEA is even slower than in the full theory. We conclude that an effective bosonic theory that includes fermionic exchange effects overestimates EEA efficiency in encapsulated $\mathrm{MoS}_{2}$ by a factor 2 . This is partly remedied by neglecting fermionic exchange as well due to a compen- 


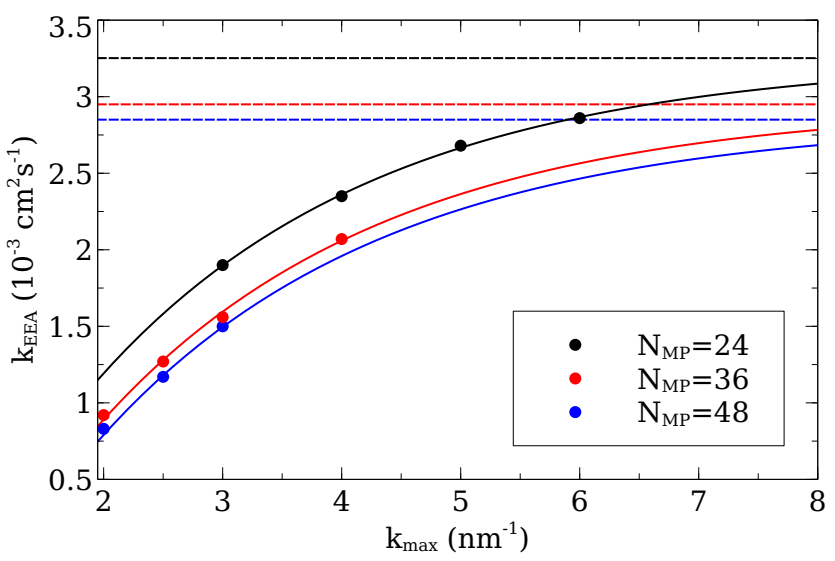

FIG. S6. Dependence of EEA coefficient in hBN-encapsulated $\mathrm{MoS}_{2}$ at $T=300 \mathrm{~K}$ on the Brillouin-zone sampling. We compare Monkhorst-Pack grids with $N_{\mathrm{MP}} \times N_{\mathrm{MP}} \times 1$ grid points for different radii $k_{\max }$ of the region taken into account around the K-point. The numerical results are shown as filled circles, while analytic fitting curves of the form $f(x)=a-$ $\exp (-b \cdot(x-c))$ are represented by solid lines. The dashed lines correspond to the asymptotic EEA coefficient obtained from the analytic functions for large radii.

sation between the different exchange effects.

\section{Brillouin zone sampling and convergence}

The numerical simulation of EEA involves several steps: the diagonalization of the BSE (2) to obtain twoparticle energies and wave functions, the calculation of exciton-exciton interaction matrix elements (5) and the propagation of the EOM for exciton populations (4) and (7). To this end, an appropriate sampling of the Brillouin zone has to be applied, where the number of grid points is constrained by the high-dimensionality of the problem. As explained in the manuscript, we focus on Bloch states in the K-valley, using a Monkhorst-Pack grid to sample the Brillouin zone in a circle with radius $k_{\max }$ around the K-point. Most results are based on a $36 \times 36 \times 1$-grid and $k_{\max }=4 \mathrm{~nm}^{-1}$, propagating the EOM until $t_{\max }=20$ ps to extract EEA coefficients $k_{\mathrm{EEA}}$ via the analytic fit formula $n_{\mathrm{X}}(t)=n_{\mathrm{X}, 0}\left(1+n_{\mathrm{X}, 0} k_{\mathrm{EEA}} t\right)^{-1}$. One exception is the time dependence of the total exciton density $n_{\mathrm{X}}(t)$ shown in Fig.2(b) for illustrative purposes. Here, we used a $24 \times 24 \times 1$-grid and $k_{\max }=3 \mathrm{~nm}^{-1}$ to reduce the numerical effort for propagating the EOM until $t_{\max }=500$ ps.

The convergence of results with respect to Brillouin zone sampling is shown in Fig. S6 for hBN-encapsulated $\mathrm{MoS}_{2}$ at $T=300 \mathrm{~K}$. The convergence with respect to $k_{\max }$ is slow, but can be well extrapolated by analytic fitting curves with exponential asymptotics. Note that the distance between $\mathrm{K}$ and $\mathrm{K}^{\prime}$ is $13.3 \mathrm{~nm}^{-1}$ for the given lattice constant. We find that results are almost converged with respect to k-point density for a $36 \times 36 \times 1$-grid. From the

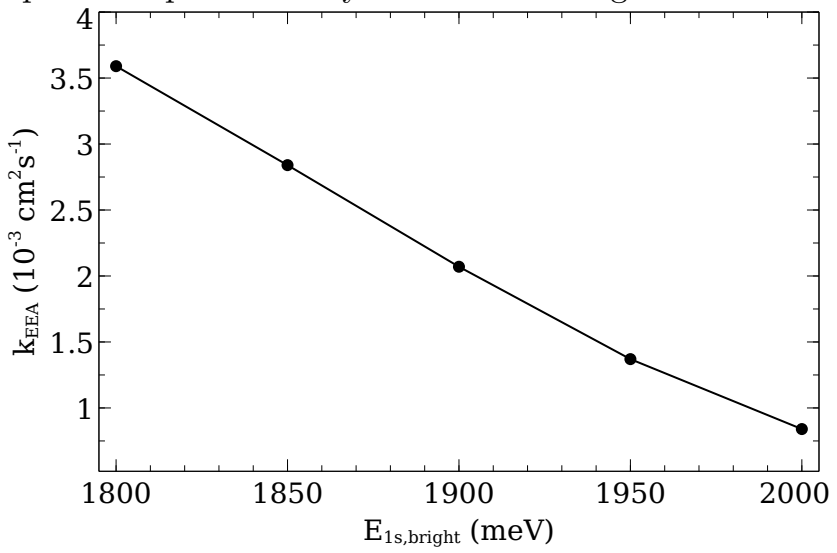

FIG. S7. Dependence of EEA coefficient in hBN-encapsulated $\mathrm{MoS}_{2}$ at $T=300 \mathrm{~K}$ on the energy of the exciton ground state $E_{1 \mathrm{~s} \text {,bright }}$, which corresponds to the like-spin exciton at the K-point with zero total momentum (so-called A-exciton).

analysis of asymptotics, we estimate a converged value $k_{\mathrm{EEA}}=2.8 \times 10^{-3} \mathrm{~cm}^{2} \mathrm{~s}^{-1}$, which is about $35 \%$ larger than the value obtained with our standard grid.

\section{E. Influence of 1s-exciton energy}

The dependence of the EEA coefficient on the groundstate exciton energy $E_{1 \text { s, bright }}$ is shown in Fig. [S7. In the manuscript, $E_{1 \mathrm{~s}, \text { bright }}=1900 \mathrm{meV}$ is used. An increase (decrease) of $E_{1 \mathrm{~s} \text {, bright }}$ corresponds to an increase (decrease) of the average energy of high-energy target states for the EEA process, see Fig. 2(a) and (c). As a trend, we find that EEA becomes more efficient for smaller $E_{1 \mathrm{~s}, \text { bright }}$. Since in this situation the target states move to lower energies, it is equivalent to a hypothetical movement of the third conduction band upwards relative to the first conduction band, which could be due to uncertainties in the underlying first-principle calculation.

\section{F. Influence of phenomenological damping}

We compare the time evolution of the total exciton density $n_{\mathrm{X}}$ for the phenomenological damping used in the manuscript $(\Gamma=50 \mathrm{meV})$ with a calculation using $\Gamma=25 \mathrm{meV}$ in Fig. S8. The Brillouin zone is sampled with our standard k-mesh. We find a weak dependence on $\Gamma$ in the range of several percent. 


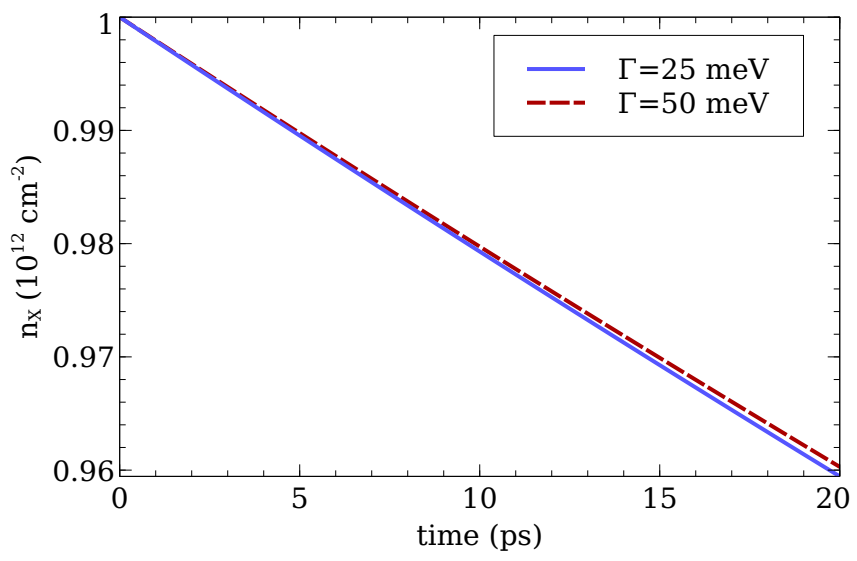

FIG. S8. Time dependence of the total exciton density $n_{\mathrm{X}}$ in hBN-encapsulated $\mathrm{MoS}_{2}$ at $T=300 \mathrm{~K}$ for two different phenomenological damping constants $\Gamma$, see Eq. (4) in the manuscript. The EEA coefficients according to Eq. (8) are $k_{\mathrm{EEA}}=2.07 \times 10^{-3} \mathrm{~cm}^{2} \mathrm{~s}^{-1}$ for $\Gamma=50 \mathrm{meV}$ and $k_{\mathrm{EEA}}=$ $2.11 \times 10^{-3} \mathrm{~cm}^{2} \mathrm{~s}^{-1}$ for $\Gamma=25 \mathrm{meV}$. 